\title{
Defects, super-Poincaré line bundle and fermionic T-duality
}

\author{
Shmuel Elitzur, ${ }^{a}$ Boaz Karni, ${ }^{a}$ Eliezer Rabinovici ${ }^{a, c}$ and Gor Sarkissian ${ }^{a, b}$ \\ ${ }^{a}$ The Racah Institute of Physics, The Hebrew University, \\ Givat Ram, 91904 Jerusalem, Israel \\ ${ }^{b}$ Department of Theoretical Physics, Yerevan State University, \\ Alex Manoogian 1, 0025 Yerevan, Armenia \\ ${ }^{c}$ CERN, \\ 1211 Geneva 23, Switzerland \\ E-mail: elitzur@vms.huji.ac.il, boazkarni@gmail.com, \\ eliezer@vms.huji.ac.il, gor.sarkissian@ysu.am
}

ABSTRACT: Topological defects are interfaces joining two conformal field theories, for which the energy momentum tensor is continuous across the interface. A class of the topological defects is provided by the interfaces separating two bulk systems each described by its own Lagrangian, where the two descriptions are related by a discrete symmetry. In this paper we elaborate on the cases in which the discrete symmetry is a bosonic or a fermionic Tduality. We review how the equations of motion imposed by the defect encode the general bosonic T- duality transformations for toroidal compactifications. We generalize this analysis in some detail to the case of topological defects allowed in coset CFTs, in particular to those cosets where the gauged group is either an axial or vector $\mathrm{U}(1)$. This is discussed in both the operator and Lagrangian approaches. We proceed to construct a defect encoding a fermionic T-duality. We show that the fermionic T-duality is implemented by the SuperPoincaré line bundle. The observation that the exponent of the gauge invariant flux on a defect is a kernel of the Fourier-Mukai transform of the Ramond-Ramond fields, is generalized to a fermionic T-duality. This is done via a fiberwise integration on supermanifolds.

KeYwords: Supersymmetry and Duality, String Duality

ARXIV EPRINT: 1301.6639 


\section{Contents}

1 Introduction 1

2 Topological defects and bosonic T-duality 4

2.1 Preliminaries 4

2.2 Factorized T-duality in non-linear sigma model 6

$\begin{array}{ll}2.3 & \text { Dualities of toroidal compactifications }\end{array}$

$\begin{array}{lll}2.4 & \text { Defects and Fourier-Mukai transform } & 10\end{array}$

3 Defects between vectorially and axially gauged WZW models 11

$\begin{array}{lll}3.1 & \text { Geometry and flux of the defects gluing axially-vectorially gauged models } & 11\end{array}$

$\begin{array}{lll}3.2 & \text { Duality defect for the parafermion disc } \mathrm{SU}(2) / \mathrm{U}(1) & 14\end{array}$

3.3 Axial-vectorial defects as operators in the parafermion Hilbert space 16

4 Fermionic T-duality $\quad 17$

$\begin{array}{lll}4.1 & \text { Pseudodifferential forms integration } & 18\end{array}$

$\begin{array}{lll}4.2 & \text { Review of the fermionic T-duality } & 18\end{array}$

4.3 Defects implementing the fermionic T-duality and the Super Poincaré line $\begin{array}{ll}\text { bundle } & 19\end{array}$

4.4 Super Fourier-Mukai transform 21

5 Discussion $\quad 21$

A Solution of the defect equations of motion in the bosonic case 22

B Geometry of the vector-axial duality defects for $\mathrm{SU}(2) / \mathrm{U}(1)$ cosets 23

$\begin{array}{ll}\text { C Some super geometry } & 25\end{array}$

D Solution of the defect equations of motion in the fermionic case $\quad 27$

\section{Introduction}

Interfaces in two-dimensional conformal field theories are playing a role in various topics, see e.g. [1-33].

Interfaces are oriented lines separating two different quantum filed theories. In this paper we consider special class of interfaces, for which the energy-momentum tensor is continuous across the defect. Denoting the left- and right- moving energy-momentum tensors of the two theories by $T^{1}, T^{2}$, and $\bar{T}^{1}, \bar{T}^{2}$, this condition takes the form:

$$
T^{(1)}=T^{(2)}, \quad \bar{T}^{(1)}=\bar{T}^{(2)}
$$


Inserting a defect/interface in the path integral is equivalent in the operator language to the insertion of an operator $D$ which maps the Hilbert space of CFT 1 to that of CFT 2 . Thus a defect can be described by such an operator. Condition (1.1) should be considered as implying also that the corresponding operator $D$ commutes with the Virasoro modes:

$$
D L_{m}^{1}=L_{m}^{2} D \quad \text { and } \quad D \bar{L}_{m}^{1}=\bar{L}_{m}^{2} D
$$

where $L_{m}^{i}$ and $\bar{L}_{m}^{i}$ act on the state space $\mathcal{H}_{i}, i=1,2$, and therefore the interface can be continuously deformed without affecting the value of correlators as long it does not cross any field insertion point. These interfaces are called topological defects [9]. Topological defects have the following properties.

- Two topological defects can be moved and merged with each other to create a new defect. In the operator language the defect fusion corresponds to the composition of the defect operators $[2-4,10]$.

- Similarly a topological defect can be moved to the boundary and fused with it, producing new boundary conditions $[3,8,11]$. The new boundary state is given by the action of the defect operator on the boundary state. Remembering that in String theory boundary states correspond to D-branes, one arrives to the conclusion that topological defects induce D-brane transformations. On the other hand D-branes are classified by their Ramond-Ramond or K-theory charges. Therefore topological defects should induce also transformations in cohomology or K-theory groups. It is expected that this transform should be of the Fourier-Mukai type [14, 16, 17, 25, 29].

Let us now take a closer look at the equations (1.1) and (1.2).

As follows from the above discussion $D$ maps an eigenstate $|a\rangle$ of the $L_{0}^{1}$ to the eigenstate of $L_{0}^{2}$, with the same eigenvalue, if $|a\rangle$ is not in the null space of $D$. Hence the left and right Hamiltonians of the two theories coincide on the pair of states $(|a\rangle, D|a\rangle)$, where $|a\rangle$ is an eigenstate of the left or right Hamiltonian of the first theory belonging to $\mathcal{H}_{1} / \operatorname{Null}(D)$. Thus the theories admitting a topological defect to join them, have the same spectra once restricted to the subspace $\left.\{(|a\rangle, D|a\rangle)|| a\rangle \in \mathcal{H}_{1} / \operatorname{Null}(D)\right\}$.

In this paper we will analyze the defects also in the Lagrangian formalism. In the Lagrangian approach to defects, one has besides the bulk equation of motion, also defect equations of motion $[15,17]$. The defect equations of motion schematically have the form $F\left(\Phi_{1}, \Phi_{2}, \Psi\right)=0$, where $F$ is some function, $\Phi_{i}$ is a collective notation for the fields of the first and the second theories, and $\Psi$ is a collective notation for fields on the defect that are not inherited from the bulk. The energy-momentum tensors coincide when the defect equations of motion are imposed. Comparing this with the operatorial picture, we see that the defect equations of motion capture the information on the structure of the defect.

Presently we are not aware of a complete classification of theories that can be joined by a topological defect, aside from the necessary condition that the theories must have the same central charges. We would like, however, to mention some typical situations where that is possible. 
The defects with a trivial null space describe theories with the same spectra. Hence these defects exploit and uncover various symmetries of the theory. In particular such defects connect different duality pictures of the same CFT, like those related by T-duality [34] and mirror symmetry $[12,13,17,24,29]$. Let us note the following properties of these defects. As explained above the defect relates eigenstates of the Hamiltonians with the coinciding values of the Hamiltonians. Therefore the defect equations of motion should produce the corresponding duality relations $[17,24]$. We demonstrate this point in this paper in some new instances.

There are several examples of theories connected by defects with a non-trivial null space. Among them are

- Scalars compactified on circles at the rationally related radii $[12,13]$.

- Torus compactifications related by $O(d, d \mid \mathbb{Q})$ semi-group transformations with rational entries [29].

- Theories related by orbifold constructions [10, 12, 17].

- different modular invariants constructed out of the same chiral data [21, 33].

In the case of defects in rational conformal field theory a relation between the corresponding theories in the terms of the underlying modular tensor categories was suggested in [30].

In this paper we

- Discuss topological defects joining axial and vector gaugings of $G / \mathrm{U}(1)$ gauged WZW models.

- Generalize the construction of defects joining theories related by bosonic T-duality to the case of superspace target spaces related by a fermionic T-duality.

The paper is organized in the following way.

In section 2 we review topological defects implementing bosonic T-duality [34].

In section 3 we construct defects between axial and vector gauging of $G / \mathrm{U}(1)$ gauged WZW models [35-38] for a general group $G$. For the case of $G=\mathrm{SU}(2)$ [39] the geometrical construction is translated to the algebraic parafermionic language. We show that for level $k$ parafermions there are $k+1$ topological defects mapping axially gauged $\mathrm{SU}(2) / \mathrm{U}(1)$ cosets to the vectorially gauged $\mathrm{SU}(2) / \mathrm{U}(1)$ coset, labeled by the integrable spin $j=0, \ldots, \frac{k}{2}$. We construct them in both the Lagrangian approach, and algebraic one, in the latter by identifying the appropriate operators in the parafermion Hilbert space. We show that the defect corresponding to $j=0$ implements $\mathbb{Z}_{k}$ orbifolding together with T-duality. These defects project $A_{j, n}$ Cardy branes in $\mathrm{SU}(2) / \mathrm{U}(1)$ coset to the $B_{j}$ branes constructed in [40].

In section 4 we study the defect performing the fermionic T-duality [41]. It is established that the defect implementing bosonic T-duality is given by the Poincaré line bundle $[14,17]$. We show that the defect inducing the fermionic T-duality is given by the fermionic generalization of the Poincaré line bundle, which we denote as Super-Poincaré line bundle. We demonstrate that the defect equations of motion reproduce the fermionic T- duality transformation rules found in [41]. Using the exponent of the gauge invariant flux on this defect as a kernel of the Fourier-Mukai transform with a pushforward map 
given by the fiberwise integration on supermanifold, we derive the transformation of the Ramond-Ramond fields under the fermionic T-duality.

In four appendices $\mathrm{A}, \mathrm{B}, \mathrm{C}$ and $\mathrm{D}$ some calculations and constructions are explained in more detail.

\section{Topological defects and bosonic T-duality}

In this section we review some basic facts concerning topological defects and their relation to T-duality. We first use the definition of the topological defects in the simple example of a scalar field compactified on a circle to demonstrate how the defect equations of motion together with the requirement to be topological reproduce the appropriate duality transformations. In the next subsection we generalize this to the factorized T-duality in non-linear sigma models with isometries. In these cases the null space of the defects is trivial and the defects are invertible. We then go on to discuss cases where the null space is non trivial. We present a defect generating a combined action of the $\mathbb{Z}_{k}$ orbifolding together with a T-duality transformation. Then we review defects implementing generators of the full $O(d, d \mid \mathbb{Z})$ duality group in the case of toroidal compactification. These defects are invertible as well. We conclude this section explaining how the T-duality transformation of the Ramond-Ramond charges can be written as the Fourier-Mukai transform with the kernel given by the exponent of the gauge invariant flux on the corresponding topological defect.

\subsection{Preliminaries}

Defects in two-dimensional quantum field theory are oriented lines separating different quantum field theories, labeled (in this paper) by 1 and 2. Conformal defects are required to satisfy [5]

$$
T^{(1)}-\bar{T}^{(1)}=T^{(2)}-\bar{T}^{(2)}
$$

Topological defects satisfy [2]

$$
T^{(1)}=T^{(2)}, \quad \bar{T}^{(1)}=\bar{T}^{(2)}
$$

Since the stress-energy tensor is a generator of diffeomorphisms, condition (2.2) implies that the defect is invariant under a distortion of the line to which it is attached. A notion of fusion between a defect and a boundary can be expected in the case of topological defects, since the latter can be moved to the boundary without changing the correlator [3].

We review the construction of an action with defects $[14,17]$. We locate the defect at the vertical line $\mathrm{S}$ defined by the condition $\sigma=0$. Denote by $\Sigma_{1}$ the left half-plane $(\sigma \leq 0)$, and by $\Sigma_{2}$ the right half-plane $(\sigma \geq 0)$, and a pair of maps $X: \Sigma_{1} \rightarrow M_{1}$ and $\tilde{X}: \Sigma_{2} \rightarrow M_{2}$, where $M_{1}$ and $M_{2}$ are the target spaces for the two quantum field theories. Suppose we have a submanifold $Q$ of the cartesian product of target spaces: $Q \subset M_{1} \times M_{2}$, with a connection one-form $A$, and a combined map:

$$
\begin{aligned}
\Phi: S & \rightarrow M_{1} \times M_{2} \\
s & \mapsto(X(s), \tilde{X}(s))
\end{aligned}
$$

which takes values in the submanifold $Q . Q$ is called the world-volume of the defect. 
In this setup we can write the action:

$$
I=\int_{\Sigma_{1}} d x^{+} d x^{-} L_{1}+\int_{\Sigma_{2}} d x^{+} d x^{-} L_{2}+\int_{S} \Phi^{*} A
$$

where

$$
\begin{aligned}
L_{1} & =E_{m n}^{(1)} \partial X^{m} \bar{\partial} X^{n}, \\
L_{2} & =E_{m n}^{(2)} \partial \tilde{X}^{m} \bar{\partial} \tilde{X}^{n}, \\
x^{ \pm} & =\tau \pm \sigma,
\end{aligned}
$$

with $E_{m n}^{(i)}$ being the components of two second rank tensors. The tensors $E^{(i)}$ are split as

$$
E^{(i)}=G^{(i)}+B^{(i)}
$$

where $G^{(i)}$ are the symmetric target space metrics of the two sigma models and $B^{(i)}$ are the corresponding NS antisymmetric two-forms.

As a warm-up exercise we work out the following simple example, when we have on both sides free scalars compactified on circles $S_{X}^{1}$ and $S_{\tilde{X}}^{1}$ of radii $R_{1}$ and $R_{2}$ :

$$
L_{1}=R_{1}^{2} \partial X \bar{\partial} X
$$

and

$$
L_{2}=R_{2}^{2} \partial \tilde{X} \bar{\partial} \tilde{X}
$$

The world-volume of the defect is a product of the target spaces $S_{X}^{1} \times S_{\tilde{X}}^{1}$ with the connection $A=-X d \tilde{X}$. The curvature of this connection is $F=d \tilde{X} \wedge d X$. This forms a Poincaré bundle $\mathcal{P}$ [42]. The equations of motion on the defect line are:

$$
\begin{aligned}
& R_{1}^{2}(\partial X-\bar{\partial} X)-\partial_{\tau} \tilde{X}=0 \\
& R_{2}^{2}(\partial \tilde{X}-\bar{\partial} \tilde{X})-\partial_{\tau} X=0
\end{aligned}
$$

For $R_{2}=\frac{1}{R_{1}},(2.11)$ and (2.12) take the form:

$$
\begin{aligned}
& R_{1}^{2}(\partial X-\bar{\partial} X)-(\partial \tilde{X}+\bar{\partial} \tilde{X})=0 \\
& (\partial \tilde{X}-\bar{\partial} \tilde{X})-R_{1}^{2}(\partial X+\bar{\partial} X)=0
\end{aligned}
$$

Equations (2.13) and (2.14) imply

$$
\begin{aligned}
& R_{1}^{2} \partial X=\partial \tilde{X} \\
& R_{1}^{2} \bar{\partial} X=-\bar{\partial} \tilde{X}
\end{aligned}
$$

which are the T-duality relations. Equations (2.15) and (2.16) also show that the defect given by the Poincaré bundle $\mathcal{P}$ for $R_{2}=\frac{1}{R_{1}}$ is topological. If this is not the case, then from equations (2.11) and (2.12) one can derive equation (2.1) and the defect is conformal, but not topological. 
One generalization that comes to mind is a defect $\mathcal{P}^{k}$ with the same world-volume but with $k$ units of the flux above: $F=k d \tilde{X} \wedge d X$. In the same way it is possible to show that this defect is topological when the radii satisfy the relation

$$
R_{1} R_{2}=k
$$

and instead of (2.15) and (2.16) one obtains:

$$
\begin{aligned}
& R_{1}^{2} \partial X=k \partial \tilde{X} \\
& R_{1}^{2} \bar{\partial} X=-k \bar{\partial} \tilde{X}
\end{aligned}
$$

These relations imply that the defect $\mathcal{P}^{k}$ combines the actions of the $Z_{k}$ orbifolding and T-duality.

All this is in agreement with $[12,13]$, where more general submanifolds $Q$ are considered. There the worldvolume $Q$ of the defect is either two dimensional with flux $F=k_{1} d \tilde{X} \wedge d X$, but allowed to wrap the product $S_{X}^{1} \times S_{\tilde{X}}^{1}$ torus $k_{2}$ times, or $Q$ is made one dimensional winding around the cycles $\left(k_{1}, k_{2}\right)$ times. Then the existence of the topological defect is proved for the radii satisfying the relations:

$$
R_{1} R_{2}=\left|\frac{k_{1}}{k_{2}}\right| \quad \text { or } \quad \frac{R_{2}}{R_{1}}=\left|\frac{k_{1}}{k_{2}}\right|
$$

where $k_{1}, k_{2} \in \mathbb{Z}$.

\subsection{Factorized T-duality in non-linear sigma model}

Let us turn to the defect description of the T-duality arising when one has action

$$
I=\int_{\Sigma} d x^{+} d x^{-} E_{m n} \partial X^{m} \bar{\partial} X^{n}
$$

on a target space with the isometry [34, 43]. Here, and in the following, repeated indices are summed over. Suppose that the coordinate $X^{1}$ is chosen in the direction of the isometry. This means that $G_{i j}$ and $B_{i j}$ do not depend on $X^{1}$. It is known that in this situation the action with the background matrix $E$ is equivalent to the action with the background matrix $\tilde{E}$, where

$$
\begin{aligned}
\tilde{E}_{11} & =\frac{1}{E_{11}} \\
\tilde{E}_{1 M} & =\frac{E_{1 M}}{E_{11}} \\
\tilde{E}_{M 1} & =-\frac{E_{M 1}}{E_{11}} \\
\tilde{E}_{M N} & =E_{M N}-\frac{E_{M 1} E_{1 N}}{E_{11}}
\end{aligned}
$$

In components one has:

$$
\begin{aligned}
\tilde{G}_{11} & =\frac{1}{G_{11}} \\
\tilde{G}_{1 M} & =\frac{B_{1 M}}{G_{11}}
\end{aligned}
$$




$$
\begin{aligned}
\tilde{B}_{1 M} & =\frac{G_{1 M}}{G_{11}} \\
\tilde{G}_{M N} & =G_{M N}-\frac{1}{G_{11}}\left(G_{M 1} G_{1 N}+B_{1 N} B_{M 1}\right) \\
\tilde{B}_{M N} & =B_{M N}-\frac{1}{G_{11}}\left(G_{M 1} B_{1 N}+G_{1 N} B_{M 1}\right)
\end{aligned}
$$

The capital latin indices run from 2 to $\operatorname{dim} M$.

The dual coordinate $\tilde{X}^{1}$ is related to the original $X^{1}$ by the relations:

$$
\partial \tilde{X}^{1}=E_{11} \partial X^{1}+E_{M 1} \partial X^{M} \quad \text { and } \quad \bar{\partial} \tilde{X}^{1}=-\left(E_{11} \bar{\partial} X^{1}+E_{1 M} \bar{\partial} X^{M}\right)
$$

The rest of the coordinates remains unchanged.

Consider the action (2.4) with a defect as in the situation above, where $M$ and $\tilde{M}$ are related by the equations (2.22), $Q$ is the correspondence space, given by the equations

$$
X^{N}=\tilde{X}^{N}, \quad N=2 \ldots \operatorname{dim} M
$$

with the connection

$$
A=-X^{1} d \tilde{X}^{1}
$$

and the curvature

$$
F=d \tilde{X}^{1} \wedge d X^{1}
$$

In this case the action (2.4) yields

$$
\begin{aligned}
E_{j 1} \partial X^{j}-E_{1 j} \bar{\partial} X^{j}-\partial_{\tau} \tilde{X}^{1} & =0 \\
E_{j N} \partial X^{j}-E_{N j} \bar{\partial} X^{j}-\tilde{E}_{j N} \partial \tilde{X}^{j}+\tilde{E}_{N j} \bar{\partial} \tilde{X}^{j} & =0, \quad N=2 \ldots \operatorname{dim} M \\
\tilde{E}_{j 1} \partial \tilde{X}^{j}-\tilde{E}_{1 j} \bar{\partial} \tilde{X}^{j}-\partial_{\tau} X^{1} & =0 .
\end{aligned}
$$

The index $j$ runs from 1 to $\operatorname{dim} M$. Additionally the conditions (2.25) imply

$$
\partial_{\tau} X^{N}=\partial_{\tau} \tilde{X}^{N}, \quad N=2 \ldots \operatorname{dim} M
$$

or in the coordinates $(2.7)$ :

$$
\partial X^{N}+\bar{\partial} X^{N}=\partial \tilde{X}^{N}+\bar{\partial} \tilde{X}^{N}, \quad N=2 \ldots \operatorname{dim} M
$$

Solving the equations (2.28), (2.29), (2.30) and (2.32) one obtains:

$$
\begin{array}{rlrl}
\bar{\partial} \tilde{X}^{N} & =\bar{\partial} X^{N} & N=2, \ldots \operatorname{dim} M \\
\partial \tilde{X}^{N} & =\partial X^{N} & N=2, \ldots \operatorname{dim} M \\
\partial \tilde{X}^{1} & =E_{11} \partial X^{1}+E_{M 1} \partial X^{M} & \\
\bar{\partial} \tilde{X}^{1} & =-\left(E_{11} \bar{\partial} X^{1}+E_{1 M} \bar{\partial} X^{M}\right) &
\end{array}
$$

The details of calculations appear in appendix A. We see that equations (2.33) coincide with the T-duality relations (2.24). Therefore the defect given by the Poincaré bundle on the correspondence space induces T-duality. 
One can check that (2.23) and (2.33) imply

$$
T=G_{i j} \partial X^{i} \partial X^{j}=\tilde{T}=\tilde{G}_{i j} \partial \tilde{X}^{i} \partial \tilde{X}^{j}
$$

and

$$
\bar{T}=G_{i j} \bar{\partial} X^{i} \bar{\partial} X^{j}=\tilde{\bar{T}}=\tilde{G}_{i j} \bar{\partial} \tilde{X}^{i} \bar{\partial} \tilde{X}^{j}
$$

which means that the defect is topological.

In this general set-up one can also consider the defect with the same world-volume given by equations (2.25) but with the flux

$$
F=k d \tilde{X}^{1} \wedge d X^{1}
$$

Repeating the calculations above one can show that this defect is topological if $E$ and $\tilde{E}$ are related by the equations

$$
\begin{aligned}
\tilde{E}_{11} & =\frac{k^{2}}{E_{11}} \\
\tilde{E}_{1 M} & =\frac{k E_{1 M}}{E_{11}} \\
\tilde{E}_{M 1} & =-\frac{k E_{M 1}}{E_{11}} \\
\tilde{E}_{M N} & =E_{M N}-\frac{E_{M 1} E_{1 N}}{E_{11}}
\end{aligned}
$$

Again the effects of the $Z_{k}$ orbifolding of the first coordinate and the T-duality are combined.

All this can be generalized to T-dualizing of several coordinates. Suppose we T-dualize the first $n$ coordinates, indexed by Greek letters. The matrix $E$ is broken to four pieces:

$$
E=\left(\begin{array}{cc}
E_{\alpha \beta} & E_{\alpha N} \\
E_{M \beta} & E_{M N}
\end{array}\right)
$$

The transformed background has the form

$$
\tilde{E}=\left(\begin{array}{cc}
E_{\alpha \beta}^{-1} & E_{\alpha \beta}^{-1} E_{\beta N} \\
-E_{M \alpha} E_{\alpha \beta}^{-1} & E_{M N}-E_{M \alpha} E_{\alpha \beta}^{-1} E_{\beta N}
\end{array}\right)
$$

Now we should consider the defect, with the world-volume

$$
X^{N}=\tilde{X}^{N}, \quad N=n+1, \ldots \operatorname{dim} M
$$

with the connection

$$
A=-\sum_{1}^{n} X^{\alpha} d \tilde{X}^{\alpha}
$$

and the curvature

$$
F=\sum_{1}^{n} d \tilde{X}^{\alpha} \wedge d X^{\alpha}
$$


In the same way it can be shown that for $M$ and $\tilde{M}$ related by equations (2.39) this defect is topological and implies the defect equations:

$$
\begin{array}{rlrl}
\bar{\partial} \tilde{X}^{N} & =\bar{\partial} X^{N} & N=n+1, \ldots \operatorname{dim} M \\
\partial \tilde{X}^{N}=\partial X^{N} & N=n+1, \ldots \operatorname{dim} M \\
\partial \tilde{X}^{\alpha}=E_{\beta \alpha} \partial X^{\beta}+E_{M \alpha} \partial X^{M} & & \\
\bar{\partial} \tilde{X}^{\alpha} & =-\left(E_{\alpha \beta} \bar{\partial} X^{\beta}+E_{\alpha M} \bar{\partial} X^{M}\right) &
\end{array}
$$

We have obtained again T-duality relations for several T-dualized coordinates.

\subsection{Dualities of toroidal compactifications}

Dualities of the toroidal compactification form the $O(n, n, \mathbb{Z})$ group. The generators of this group are factorized dualities, integer shifts of the flux of $B$ fields and the integer basis changes $[34,44,45]$. Defects inducing factorized dualities were discussed in the previous subsection. For completeness let us mention defects inducing the $B$-flux shift and the integer basis change symmetries.

Consider diagonal defect

$$
X^{i}=\tilde{X}^{i} \quad i=1, \ldots, \operatorname{dim} M
$$

with flux $F$. In this case equations of motion for the defect take the form:

$$
E_{j i} \partial X^{j}-E_{i j} \bar{\partial} X^{j}-\tilde{E}_{j i} \partial \tilde{X}^{j}+\tilde{E}_{i j} \bar{\partial} \tilde{X}^{j}+F_{i j} \partial_{\tau} X^{j}=0
$$

and additionally

$$
\partial X^{i}+\bar{\partial} X^{i}=\partial \tilde{X}^{i}+\bar{\partial} \tilde{X}^{i}
$$

It can be seen that if the matrices $E$ and $\tilde{E}$ differ only in the $B$ field and the difference is equal to $F$ :

$$
\begin{aligned}
\tilde{G} & =G \\
\tilde{B} & =B-F
\end{aligned}
$$

this defect is topological and implies:

$$
\begin{aligned}
\bar{\partial} \tilde{X}^{i} & =\bar{\partial} X^{i} \\
\partial \tilde{X}^{i} & =\partial X^{i}
\end{aligned}
$$

This example was considered in [17].

Another interesting example is given by a defect with world-volume given by a linear embedding:

$$
X^{i}=A_{k}^{i} \tilde{X}^{k}
$$

and with no flux. The defect equations of motion are:

$$
\left(E_{j}^{i} \partial X^{j}-E_{j}^{i} \bar{\partial} X^{j}\right) A_{k}^{i}-\tilde{E}_{j k} \partial \tilde{X}^{j}+\tilde{E}_{k j} \bar{\partial} \tilde{X}^{j}=0
$$


and additionally

$$
\partial X^{i}+\bar{\partial} X^{i}=A_{k}^{i}\left(\partial \tilde{X}^{k}+\bar{\partial} \tilde{X}^{k}\right)
$$

One can verify that if $E$ and $\tilde{E}$ satisfy the relation:

$$
\tilde{E}_{m k}=E_{j i} A_{k}^{i} A_{m}^{j}
$$

this defect is topological and the defect equations of motion are solved by

$$
\begin{aligned}
& \partial X^{i}=A_{k}^{i} \partial \tilde{X}^{k} \\
& \bar{\partial} X^{i}=A_{k}^{i} \bar{\partial} \tilde{X}^{k}
\end{aligned}
$$

For the torus compactifications the Dirac's quantization condition of the flux $F_{i j}$ and the quantization imposed on the matrix $A_{k}^{i}$ by the compactness of the defect bring to the appropriate integer $B$-flux shifts and the integer basis change transformations [34, 44, 45]. Considering multiply wrapped defects leads to the extended semi-group $O(d, d, \mathbb{Q})$ of the defects $[12,29]$

\subsection{Defects and Fourier-Mukai transform}

As explained at the beginning, a topological defect can be fused with a boundary, producing a new boundary condition from the old one. On the other hand boundary conditions correspond to D-branes, which can be characterized by their Ramond-Ramond charges or more precisely by an element of the K-theory. Therefore an action of the defect on the RR charges and K-theory elements should be defined. The form of this action on RR charges turns out to be connected to the flux on the corresponding defect. Mathematically this flux serves as a kernel of an operation known as Fourier-Mukai transformation [46, 47].

Consider for example the T-duality transformation of the Ramond-Ramond fields.

It is found in [48] that the T-duality transformation of the Ramond-Ramond fields RR fields $[49,50]$ of the theory on $T^{n} \times M$ and those of the T-dual theory on $\hat{T}^{n} \times M$ are related by a Fourier-Mukai transform:

$$
\hat{\mathcal{G}}=\int_{T^{n}} e^{\hat{B}-B+\sum_{i=1}^{n} d \hat{t}_{i} \wedge d t^{i}} \mathcal{G}
$$

Here $B$ is the Neveu-Schwarz $B$-field and $\mathcal{G}=\sum_{p} \mathcal{G}_{p+2}$ is the sum of gauge invariant RR field strength where the sum is over $p=0,2,4, \ldots$ for Type IIA and $p=-1,1,3, \ldots$ for Type IIB. The integrand in (2.54) is considered as a form on the space $M \times T^{n} \times \hat{T}^{n}$ and the fiberwise integration $\int_{T^{n}}$, maps forms on $M \times T^{n} \times \hat{T}^{n}$ to forms on $M \times \hat{T}^{n}$. The integral operates on the forms of the highest degree $n$ in $d t_{i}$ and sets to zero forms of lower degree in $d t_{i}[51]$ :

$$
\begin{aligned}
f\left(x, \hat{t}_{i}, t^{i}\right) p^{*} \omega & \wedge d t_{i_{1}} \wedge \ldots d t_{i_{r}} & \mapsto 0, & r<n \\
f\left(x, \hat{t}_{i}, t^{i}\right) p^{*} \omega & \wedge d t_{1} \wedge \ldots d t_{n} & \mapsto \omega \int_{T^{n}} f\left(x, \hat{t}_{i}, t^{i}\right) d t_{1} \ldots d t_{n} &
\end{aligned}
$$

Here $p$ is the projection $M \times T^{n} \times \hat{T}^{n} \rightarrow M \times \hat{T}^{n}, \omega$ is a form on $M \times \hat{T}^{n}, f\left(x, \hat{t}_{i}, t^{i}\right)$ is an arbitrary function and $x$ denotes a point in $M$. The fiberwise integration (2.55) is actually 
the Berezin integration, which is not surprising when one remembers that the one-forms $d t_{i}$ anticommute.

Note that the kernel of the Fourier-Mukai transform (2.54) is indeed the exponent of the gauge invariant combination of the $B$ fields and the flux of the T-duality defect

$$
e^{\mathcal{F}}=e^{\hat{B}-B+\sum_{i=1}^{n} d \hat{t}_{i} \wedge d t^{i}}
$$

Let us check that in the simple case of T-dualizing of one coordinate and without a $B$ field, that formula (2.54) yields the known map of $D p$ to $D(p \pm 1)$ branes. In this case eq. (2.54) takes the form

$$
\hat{\mathcal{G}}=\int_{S^{1}} \mathcal{G} e^{d t \wedge d \hat{t}}=\int_{S^{1}} \mathcal{G}(1+d t \wedge d \hat{t})
$$

Suppose that the $D p$-brane is transverse to the coordinate $t$ and therefore the volume- form $\mathcal{G}$ does not contain $d t$. In this case (2.57) according to (2.55) implies

$$
\hat{\mathcal{G}}=\mathcal{G} \wedge d \hat{t}
$$

and thus $\hat{\mathcal{G}}$ describes $D(p+1)$-brane as expected. Now consider the case when $D p$-brane contains the coordinate $t$ and therefore the volume-form $\mathcal{G}$ has the form $\mathcal{G}=\omega \wedge d t$. In this case eq. (2.57) yields

$$
\hat{\mathcal{G}}=\omega
$$

and represents $D(p-1)$-branes again in agreement with T-duality.

\section{Defects between vectorially and axially gauged WZW models}

In this section we construct topological defects mapping the axially gauged $\frac{G}{\mathrm{U}(1)}$ axial $\mathrm{WZW}$ model to the vectorially gauged $\frac{G}{\mathrm{U}(1)}$ vectorial WZW model for a general group $G$. For the case $G=\mathrm{SU}(2)$ we analyze the corresponding operators acting in the Hilbert space of parafermions and find that for the level $k$ parafermions there are $k+1$ such topological defects, labeled by the integrable spin $j=0, \ldots, \frac{k}{2}$. This is another example of the case of a non trivial null space for the defect. The object is to realize these defects in the Lagrangian approach as a line separating axially and vectorially gauged WZW models. This problem is solved in this section. First we present the geometrical ansatz for the defects (formula (3.14) below) and check that it leads to the action that glues axially and vectorially gauged models. Then we study in detail the defect given by $j=0$ and show that it coincides with the defect with the flux (2.36), studied in the previous section, and implements $\mathbb{Z}_{k}$ orbifolding together with the T-duality. In the rest of the section we construct defects as operators in the Hilbert space of the parafermions. In appendix B, we calculate the overlap of these operators with the eigen-position state and show that they have the geometry of the ansatz (3.14).

\subsection{Geometry and flux of the defects gluing axially-vectorially gauged models}

The action of the gauged WZW model is [35-38]:

$$
S^{G / H}(g, A)=S^{\mathrm{WZW}}+S^{\text {gauge }},
$$


where

$$
\begin{aligned}
S^{\mathrm{WZW}}(g) & =\frac{k}{4 \pi} \int_{\Sigma} \operatorname{Tr}\left(\partial_{+} g \partial_{-} g^{-1}\right) d x^{+} d x^{-}+\frac{k}{4 \pi} \int_{B} \frac{1}{3} \operatorname{tr}\left(g^{-1} d g\right)^{3} \\
& \equiv \frac{k}{4 \pi}\left[\int_{\Sigma} d x^{+} d x^{-} L^{\mathrm{kin}}+\int_{B} \omega^{\mathrm{WZ}}\right], \\
S^{\text {gauge }} & =\frac{k}{2 \pi} \int_{\Sigma} L_{v}^{\text {gauge }} d x^{+} d x^{-}, \\
L_{v}^{\text {gauge }}(g, A) & =\operatorname{tr}\left[-g^{-1} \partial_{+} g A_{-}+\partial_{-} g g^{-1} A_{+}+A_{-} g^{-1} A_{+} g-A_{+} A_{-}\right] .
\end{aligned}
$$

Here $H$ is subgroup of $G, g \in G$ and $B$ is a 3-manifold such that $\partial B=\Sigma$ and $A$ is a gauge field taking values in the $H$ Lie algebra.

Using the Polyakov-Wiegmann identities:

$$
\begin{aligned}
L^{\mathrm{kin}}(g h) & =L^{\mathrm{kin}}(g)+L^{\mathrm{kin}}(h)-\left(\operatorname{Tr}\left(g^{-1} \partial_{+} g \partial_{-} h h^{-1}\right)+\operatorname{Tr}\left(g^{-1} \partial_{-} g \partial_{+} h h^{-1}\right)\right), \\
\omega^{\mathrm{WZ}}(g h) & =\omega^{\mathrm{WZ}}(g)+\omega^{\mathrm{WZ}}(h)-\mathrm{d}\left(\operatorname{Tr}\left(g^{-1} \mathrm{~d} g \mathrm{~d} h h^{-1}\right)\right)
\end{aligned}
$$

it is possible to verify that the action (3.1) is invariant under the gauge transformation:

$$
g \rightarrow h g h^{-1}, \quad A \rightarrow h A h^{-1}+d h h^{-1}
$$

for $h: \Sigma \rightarrow H$. This is a vectorially gauged model.

For the case of $H=\mathrm{U}(1)$ considered here there exists the system is axially gauge invariant under the transformations

$$
g \rightarrow h g h, \quad A \rightarrow A+d h h^{-1}
$$

for $h: \Sigma \rightarrow \mathrm{U}(1)$. In the axially gauged model the gauge field dependent term is

$$
L_{a}^{\text {gauge }}(g, A)=\operatorname{tr}\left[g^{-1} \partial_{+} g A_{-}+\partial_{-} g g^{-1} A_{+}-A_{-} g^{-1} A_{+} g-A_{+} A_{-}\right] .
$$

There are several steps needed in order to write a well defined action on the defect, with an image in the submanifold $Q \subseteq G \times G$

$$
S \rightarrow G \times G: s \mapsto\left(g_{1}(s), g_{2}(s)\right) \in Q,
$$

with a defect line $S$ separating vectorially and axially gauged models, in the presence of a WZW form [14].

First, there should exist a two-form $\varpi$ satisfying the relation

$$
d \varpi\left(g_{1}, g_{2}\right)=\left.\omega^{\mathrm{WZ}}\left(g_{1}\right)\right|_{Q}-\left.\omega^{\mathrm{WZ}}\left(g_{2}\right)\right|_{Q}
$$

Second, one should introduce an auxiliary disc $D$ satisfying the conditions:

$$
\partial B_{1}=\Sigma_{1} \cup D \quad \text { and } \quad \partial B_{2}=\Sigma_{2} \cup \bar{D} \text {, }
$$

where the unions are such that $\partial \Sigma_{1}=\partial D=S$ and $\partial \Sigma_{2}=\partial \bar{D}=\bar{S}$, but the orientations of the gluing are opposite. 
The fields $g_{1}$ and $g_{2}$ are extended to this disc while holding the condition (3.10). After this preparations the topological part of the action takes the form [14]

$$
S^{\text {top-def }}=\frac{k}{4 \pi} \int_{B_{1}} \omega^{\mathrm{WZ}}\left(g_{1}\right)+\frac{k}{4 \pi} \int_{B_{2}} \omega^{\mathrm{WZ}}\left(g_{2}\right)-\frac{k}{4 \pi} \int_{D} \varpi\left(g_{1}, g_{2}\right)
$$

One should choose an appropriate $Q$. One of the requirements is that $Q$ would be invariant under the vector and axial transformations. We suggest the following ansatz:

$$
\left(g_{1}, g_{2}\right)=\left(C_{\mu} p, L_{1} p L_{2}\right)
$$

Here $p \in G, L_{1} \in \mathrm{U}(1), L_{2} \in \mathrm{U}(1)$ and $C_{\mu}$ is a conjugacy class

$$
C_{\mu}=l e^{2 i \pi \mu / k} l^{-1}, \quad l \in G
$$

where $\mu \equiv \boldsymbol{\mu} \cdot \boldsymbol{H}$ is a highest weight representation integrable at level $k$, taking value in the Cartan subalgebra of the $G$ Lie algebra. This condition is a consequence of global issues [14]. Note that under the full gauge transformation

$$
g_{1} \mapsto h_{1} g_{1} h_{1}^{-1} \quad \text { and } \quad g_{2} \mapsto h_{2} g_{2} h_{2}
$$

the parameters in (3.14) transform as

$$
\begin{aligned}
C_{\mu} & \mapsto h_{1} C_{\mu} h_{1}^{-1} \\
p & \mapsto h_{1} p h_{1}^{-1} \\
L_{1} & \mapsto L_{1} h_{1}^{-1} h_{2} \\
L_{2} & \mapsto L_{2} h_{1} h_{2}
\end{aligned}
$$

Using the Polyakov-Wiegamann identity (3.6) one can check that the condition (3.11) is satisfied with the following two-form

$$
\begin{aligned}
\varpi\left(C_{\mu}, p, L_{1}, L_{2}\right)= & \omega_{\mu}\left(C_{\mu}\right)-\operatorname{Tr}\left(C_{\mu}^{-1} d C_{\mu} d p p^{-1}\right)+\operatorname{Tr}\left(p^{-1} d p d L_{2} L_{2}^{-1}\right)+ \\
& +\operatorname{Tr}\left(L_{1}^{-1} d L_{1} d p p^{-1}\right)+\operatorname{Tr}\left(L_{1}^{-1} d L_{1} p d L_{2} L_{2}^{-1} p^{-1}\right)-\operatorname{Tr}\left(L_{1}^{-1} d L_{1} L_{2}^{-1} d L_{2}\right)
\end{aligned}
$$

where $\omega_{\mu}\left(C_{\mu}\right)=\operatorname{Tr}\left(l^{-1} d l e^{2 i \pi \mu / k} l^{-1} d l e^{-2 i \pi \mu / k}\right)$. Now the full action can be written as

$$
S^{\mathrm{A}-\mathrm{V}}=S^{\mathrm{kin}-\mathrm{def}}+S^{\text {gauge-def }}+S^{\mathrm{top}-\operatorname{def}}
$$

here

$$
S^{\mathrm{kin}-\mathrm{def}}=\frac{k}{4 \pi} \int_{\Sigma_{1}} d x^{+} d x^{-} L^{\mathrm{kin}}\left(g_{1}\right)+\frac{k}{4 \pi} \int_{\Sigma_{2}} d x^{+} d x^{-} L^{\mathrm{kin}}\left(g_{2}\right)
$$

and

$$
S^{\text {gauge-def }}=\frac{k}{2 \pi} \int_{\Sigma_{1}} L_{v}^{\text {gauge }}\left(g_{1}, A_{1}\right) d x^{+} d x^{-}+\frac{k}{2 \pi} \int_{\Sigma_{2}} L_{a}^{\text {gauge }}\left(g_{2}, A_{2}\right) d x^{+} d x^{-}
$$

It is cumbersome but possible to check that the action (3.19) is invariant the gauge transformations:

$$
\begin{array}{lll}
g_{1} \mapsto h_{1} g_{1} h_{1}^{-1}, & A_{1} \mapsto A_{1}+d h_{1} h_{1}^{-1} \\
g_{2} \mapsto h_{2} g_{2} h_{2}, & A_{2} \mapsto A_{2}+d h_{2} h_{2}^{-1}
\end{array}
$$

where $h_{1}: \Sigma_{1} \rightarrow \mathrm{U}(1)$ and $h_{2}: \Sigma_{2} \rightarrow \mathrm{U}(1)$. 


\subsection{Duality defect for the parafermion disc $\mathrm{SU}(2) / \mathrm{U}(1)$}

Specialize now to the case of $G=\mathrm{SU}(2)$ [39].

We write the group elements using the Euler coordinates:

$$
g=e^{i \chi \frac{\sigma_{3}}{2}} e^{i \theta \sigma_{1}} e^{i \varphi \frac{\sigma_{3}}{2}}=e^{i(\tilde{\phi}+\phi) \frac{\sigma_{3}}{2}} e^{i \theta \sigma_{1}} e^{i(\tilde{\phi}-\phi) \frac{\sigma_{3}}{2}}
$$

The ranges of the variables are $0 \leq \theta \leq \frac{\pi}{2}, \quad 0 \leq \varphi \leq 2 \pi, \quad 0 \leq \chi \leq 4 \pi, \quad-\pi \leq \phi, \tilde{\phi} \leq \pi$.

The axially gauged model $\frac{\mathrm{SU}(2)}{\mathrm{U}(1)}$ axial is derived by the gauging of the $\mathrm{U}(1)$ symmetry corresponding to shifting of $\tilde{\phi}$ and has the target space $M_{A}$ with the following metric and dilaton field [34, 40]:

$$
\begin{aligned}
d s^{2} & =k\left(d \theta^{2}+\tan ^{2} \theta d \phi^{2}\right) \\
e^{\Phi} & =\frac{g_{s}}{\cos \theta} \\
\phi & \sim \phi+2 \pi
\end{aligned}
$$

Using the T-duality rules of the previous section one can see that T-dual background to the axially gauged model is

$$
\begin{aligned}
\tilde{d s}^{2} & =k\left(d \tilde{\theta}^{2}+\frac{d \tilde{\phi}^{2}}{\tan ^{2} \tilde{\theta}}\right) \\
e^{\tilde{\Phi}} & =\frac{g_{s}}{\sqrt{k} \sin \tilde{\theta}} \\
\tilde{\phi} & \sim \tilde{\phi}+\frac{2 \pi}{k}
\end{aligned}
$$

Vectorially gauged model $\frac{\mathrm{SU}(2)}{\mathrm{U}(1)}$ vec is derived by the gauging of the $\mathrm{U}(1)$ symmetry corresponding to the shifting of $\phi$ and has the target space $M_{V}$ with the metric and the dilaton:

$$
\begin{aligned}
\tilde{d s}^{2} & =k\left(d \tilde{\theta}^{2}+\frac{d \tilde{\phi}^{2}}{\tan ^{2} \tilde{\theta}}\right) \\
e^{\tilde{\Phi}} & =\frac{g_{s}}{\sin \tilde{\theta}} \\
\tilde{\phi} & \sim \tilde{\phi}+2 \pi
\end{aligned}
$$

Comparing (3.25) and (3.26) one can see that the background T-dual to the axially gauged model is the $Z_{k}$ orbifold of the vectorially gauged model.

According to the results of the previous section the world-volume of the T-duality defect $D_{A}^{T}$ between backgrounds (3.24) and (3.25) is the submanifold $\theta=\tilde{\theta}$ of the product $M_{V} \times M_{A}$ with the flux $F=d \phi \wedge d \tilde{\phi}$. The defects between backgrounds (3.24) and (3.26) $D_{V-A}$ has the same world volume but the flux is $F=k d \phi \wedge d \tilde{\phi}$.

Consider the defects given by equation (3.14). The conjugacy class takes the form $C_{j}=l e^{\frac{2 \pi i j \sigma_{3}}{k}} l^{-1}, j=0, \frac{1}{2} \ldots \frac{k}{2}$, (since we are working in the specific case of $G=\mathrm{SU}(2)$, the general subscript $\mu$ was changed to $j$, which is standard for this group) and therefore we 
have a family of the defects labelled by $j$. Now we show that the T-duality defect above, $D_{V-A}$, corresponds to $j=0$.

Let us examine this defect in more detail. Parameterizing $L_{1}=e^{i \alpha_{1} \sigma_{3} / 2}$ and $L_{2}=$ $e^{i \alpha_{2} \sigma_{3} / 2}$ and writing $p$ using the Euler coordinates, we obtain for this special defect:

$$
\left(g_{1}, g_{2}\right)=\left(e^{i(\tilde{\kappa}+\kappa) \frac{\sigma_{3}}{2}} e^{i \theta \sigma_{1}} e^{i(\tilde{\kappa}-\kappa) \frac{\sigma_{3}}{2}}, \quad e^{i\left(\tilde{\kappa}+\kappa+\alpha_{1}\right) \frac{\sigma_{3}}{2}} e^{i \theta \sigma_{1}} e^{i\left(\tilde{\kappa}-\kappa+\alpha_{2}\right) \frac{\sigma_{3}}{2}}\right)
$$

From (3.27) it can be seen that this defect satisfies the condition $\theta=\tilde{\theta}$. To project down this defect to the product space $M_{V} \times M_{A}$ we impose gauge fixing conditions $\kappa=0$ for the first vectorially gauged model and

$$
\left(\tilde{\kappa}+\kappa+\alpha_{1}\right)+\left(\tilde{\kappa}-\kappa+\alpha_{2}\right)=0
$$

for the axially gauged model. From (3.28) one obtains:

$$
\tilde{\kappa}=-\frac{\alpha_{1}+\alpha_{2}}{2}
$$

Therefore the angles $\phi$ and $\tilde{\phi}$ of the target spaces are related to the defect parameters by equations:

$$
\begin{aligned}
\tilde{\phi}=\tilde{\kappa} & =-\frac{\alpha_{1}+\alpha_{2}}{2} \\
\phi & =\frac{\alpha_{1}-\alpha_{2}}{2}
\end{aligned}
$$

Let us evaluate the two-form (3.18). For $j=0$ it simplifies to:

$$
\begin{aligned}
\varpi\left(p, L_{1}, L_{2}\right)= & \operatorname{Tr}\left(p^{-1} d p d L_{2} L_{2}^{-1}\right)+\operatorname{Tr}\left(L_{1}^{-1} d L_{1} d p p^{-1}\right)+ \\
& +\operatorname{Tr}\left(L_{1}^{-1} d L_{1} p d L_{2} L_{2}^{-1} p^{-1}\right)-\operatorname{Tr}\left(L_{1}^{-1} d L_{1} L_{2}^{-1} d L_{2}\right)
\end{aligned}
$$

This implies

$$
\begin{aligned}
\operatorname{Tr}\left(p^{-1} d p d L_{2} L_{2}^{-1}\right) & =-\left(d \tilde{\kappa} \cos ^{2} \theta-d \kappa \sin ^{2} \theta\right) d \alpha_{2} \\
\operatorname{Tr}\left(L_{1}^{-1} d L_{1} d p p^{-1}\right) & =-d \alpha_{1}\left(d \tilde{\kappa} \cos ^{2} \theta+d \kappa \sin ^{2} \theta\right) \\
\operatorname{Tr}\left(L_{1}^{-1} d L_{1} p d L_{2} L_{2}^{-1} p^{-1}\right) & =-d \alpha_{1} d \alpha_{2}\left(\cos ^{2} \theta-\frac{1}{2}\right) \\
-\operatorname{Tr}\left(L_{1}^{-1} d L_{1} L_{2}^{-1} d L_{2}\right) & =\frac{d \alpha_{1} d \alpha_{2}}{2}
\end{aligned}
$$

Using that $\kappa=0$ and (3.29), (3.30) and (3.31) one obtains that the $\theta$ dependent terms drop and we end up with

$$
\frac{k}{4 \pi} \varpi\left(p, L_{1}, L_{2}\right)=\frac{k}{4 \pi} d \alpha_{1} d \alpha_{2}=\frac{k}{2 \pi} d \tilde{\phi} d \phi
$$

This is the flux on the defect $D_{V-A}$ and as demonstrated in section 2, this defect is topological.

It is shown in appendix B that a generic defect has a geometry given by the inequality

$$
\cos 2(\theta-\tilde{\theta}) \geq \cos \frac{4 \pi j}{k}
$$




\subsection{Axial-vectorial defects as operators in the parafermion Hilbert space}

It has been shown that the backgrounds (3.24) and (3.26) correspond to the parafermion theory, and therefore the defects above can be realized as operators in the parafermions Hilbert space.

To construct the corresponding operator one should start with the Cardy defect in the parafermion theory [2]:

$$
X_{\hat{j}, \hat{n}}=\sum_{j, n} \frac{S_{(\hat{j}, \hat{n}) ;(j, n)}^{\mathrm{PF}}}{S_{(0,0) ;(j, n)}^{\mathrm{PF}}} P_{j, n}^{\mathrm{PF}} \bar{P}_{j, n}^{\mathrm{PF}}
$$

Here $S_{(\hat{j}, \hat{n}) ;(j, n)}^{\mathrm{PF}}$ is the parafermion matrix of the modular transformation

$$
S_{(\hat{j}, \hat{n}) ;(j, n)}^{\mathrm{PF}}=\sqrt{\frac{2}{k}} S_{\hat{j} j}^{\mathrm{SU}(2)} e^{\frac{i \pi n \hat{n}}{k}}
$$

$P_{j, n}^{\mathrm{PF}}$ and $\bar{P}_{j, n}^{\mathrm{PF}}$ are projectors

$$
\begin{aligned}
& P_{j, n}^{\mathrm{PF}}=\sum_{N}|j, n, N\rangle_{0} \otimes_{1}\langle j, n, N| \\
& \bar{P}_{j, n}^{\mathrm{PF}}=\sum_{M} \overline{|j, n, M\rangle_{0}} \otimes_{1} \overline{\langle j, n, M|}
\end{aligned}
$$

where the sums over $M$ and $N$ are over orthonormal bases of the parafermion state spaces. Subscriptes 0 and 1 distinguish between the theories on the two sides of the defect. Here $j \in\left\{0, \frac{1}{2}, \ldots \frac{k}{2}\right\}$ and $n \in \mathbb{Z} / 2 k \mathbb{Z}$ satisfy the constraint $2 j+n=0 \bmod 2$. The pairs $(j, n)$ and $(k / 2-j, k+n)$ have to be identified. We need to construct a defect mapping $A$ - branes to $B$ - branes. This can be done along the lines used in [40] for the parafermion $B$-branes construction. Recall that the $\mathbb{Z}_{k}$ orbifold of the parafermion theory at level $k$ is T-dual to the original theory. To get a defect mapping $A$ - branes to $B$ - branes one should sum over $\mathbb{Z}_{k}$ images of $X_{\hat{j}, \hat{n}}$ and perform T-duality. In order to circumvent the fixed point problem, we consider the case of odd $k .{ }^{1}$ Summing over images leaves in (3.36) only the $n=0$ term and T-duality exchanges $\bar{P}_{j, n}^{\mathrm{PF}}$ with its B-type version, which can be derived in the following way. Define also corresponding projectors for $\mathrm{SU}(2)$ :

$$
\begin{aligned}
& P_{j}^{\mathrm{SU}(2)}=\sum_{N}|j, N\rangle_{0} \otimes_{1}\langle j, N| \\
& \bar{P}_{j}^{\mathrm{SU}(2)}=\sum_{M} \overline{|j, M\rangle_{0}} \otimes_{1} \overline{\langle j, M|}
\end{aligned}
$$

where the sums over $N$ and $M$ are over orthonormal bases of the $\mathrm{SU}(2)$ state spaces, and rational U(1) scalar:

$$
P_{r \pm}^{\mathrm{U}(1)}=\exp \left[ \pm \sum_{n=1}^{\infty} \frac{\alpha_{-n}^{0} \alpha_{n}^{1}}{n}\right] \sum_{l \in Z}\left|\frac{r+2 k l}{\sqrt{2 k}}\right\rangle_{0} \otimes_{1}\left\langle \pm \frac{r+2 k l}{\sqrt{2 k}}\right|
$$

\footnotetext{
${ }^{1}$ In the case of an even $k$, the primary field $\frac{k}{4}$ has the non-trivial stabilizator $\mathbb{Z}_{2}$, which requires the fixed point resolution procedure. As a consequence the formulae for branes and defects derived in this way get modified. See for details $[40,52,53]$.
} 


$$
\bar{P}_{r^{\prime} \pm}^{\mathrm{U}(1)}=\exp \left[ \pm \sum_{n=1}^{\infty} \frac{\bar{\alpha}_{-n}^{0} \bar{\alpha}_{n}^{1}}{n}\right] \sum_{l \in Z} \overline{\left| \pm \frac{r^{\prime}+2 k l^{\prime}}{\sqrt{2 k}}\right\rangle_{0}} \otimes_{1} \overline{\left\langle\frac{r^{\prime}+2 k l^{\prime}}{\sqrt{2 k}}\right|}
$$

Using the decomposition of $\mathrm{SU}(2)_{k}$ as a product of parafermion and scalar theories one can write

$$
\bar{P}_{j}^{\mathrm{SU}(2)}=\sum_{r} \bar{P}_{j, r}^{\mathrm{PF}} \bar{P}_{r+}^{\mathrm{U}(1)}
$$

To define the T-dual projector $\overline{B P}_{j, n}^{\mathrm{PF}}$ we rotate the $\mathrm{SU}(2)$ projector $\bar{P}_{j}^{\mathrm{SU}(2)}$ with operator $e^{i \pi \bar{J}_{0}^{1}}$, satisfying

$$
e^{i \pi \bar{J}_{0}^{1}} \bar{J}_{0}^{3} e^{-i \pi \bar{J}_{0}^{1}}=-\bar{J}_{0}^{3}
$$

and afterwards decompose it again as a product of the parafermion and scalar theories:

$$
1 \otimes e^{i \pi \bar{J}_{0}^{1}} \bar{P}_{j}^{\mathrm{SU}(2)}=\sum_{r} \overline{B P}_{j, r}^{\mathrm{PF}} \bar{P}_{r-}^{\mathrm{U}(1)}
$$

Combining the orbifolding and the $\mathrm{T}$ duality procedures results is:

$$
Y_{\hat{j}}^{A B}=\sqrt{k} \sum_{j} \frac{S_{\hat{j}, j}^{\mathrm{SU}(2)}}{S_{0, j}^{\mathrm{SU}(2)}} P_{j, 0}^{\mathrm{PF}} \overline{B P}_{j, 0}^{\mathrm{PF}}
$$

It is shown in the appendix that in the large $k$ limit $Y_{j}^{A B}$ has the geometry given with the overlap

$$
\begin{aligned}
& \left\langle\theta, \phi\left|Y_{\hat{j}}^{A B}\right| \tilde{\theta}, \tilde{\phi}\right\rangle \sim \\
& \sim \frac{k}{\pi^{2}} \int_{|2 \theta-2 \tilde{\theta}|}^{2 \theta+2 \tilde{\theta}} \frac{\Theta(\cos \gamma-\cos 2 \hat{\psi})}{\sqrt{\cos \gamma-\cos 2 \hat{\psi}}} \frac{\sin \gamma d \gamma}{\sqrt{[\cos \gamma-\cos 2(\theta+\tilde{\theta})][\cos 2(\theta-\tilde{\theta})-\cos \gamma]}}
\end{aligned}
$$

where $\hat{\psi}=\frac{(2 \hat{j}+1) \pi}{k+2}$ and $\Theta$ is the Heavyside step function. Eq. (3.48) shows that the worldvolume of the defect should satisfy the inequality

$$
\cos 2(\theta-\tilde{\theta}) \geq \cos \hat{\psi}
$$

which in the large $k$ limit coincides with the inequality (3.35), defining the geometry of a generic defect.

Note that in the defect $Y_{0}^{A B}$, the relation of the elements of the matrix of the modular transformation drops, and it is a sum of projectors, projecting down to the $n=0$ subspace and performing T-duality, thus mapping the $A_{j, n}$ Cardy branes to the $B_{j}$ branes constructed in [40]. For generic $\hat{j}$ one derives a linear combination of the $B_{l}$ branes with coefficients given by the fusion numbers $N_{\hat{j} j}^{l}$.

\section{Fermionic T-duality}

In this section we show how do defects generate T-duality on fermionic coordinates. We show here that the fermionic T-duality is implemented by the defect, given by the fermionic 
analogue of the Poincaré line bundle, which we call Super-Poincaré line bundle. This defect is invertible.

Then we define the super Fourier-Mukai transform, as in the bosonic case, as an integral with an appropriate kernel given by the exponent of the flux of a super Poincare line bundle.

\subsection{Pseudodifferential forms integration}

The technical details can be found in appendix C, the result [54-56] is presented here. Pseudodifferential forms, defined on a supermanifold of $p$ bosonic and $q$ fermionic coordinates, are of the form

$$
f=\sum_{v, u} f_{v, u}(x, d \theta) \theta^{v} d x^{u}
$$

Where: $v=v_{1}, \ldots, v_{q} ; u=u_{1}, \ldots, u_{p} ; v_{i}, u_{i} \in 0,1 ; x=x_{1}, \ldots x_{p} ; d \theta=d \theta_{1}, \ldots d \theta_{q} ; \theta^{v}=$ $\theta_{1}^{v_{1}} \cdot \ldots \cdot \theta_{q}^{v_{q}} ; d x^{u}=d x_{1}^{u_{1}} \cdot \ldots \cdot d x_{p}^{u_{p}}$, and the sum is over all possible values of $u$ and $v$. Such an object can be integrated over the bundle on which it is defined. The integration is defined as

$$
\int_{\mathcal{B}} f=\int_{B} f_{1,1, \ldots, 1}
$$

Where $\mathcal{B}$ is the cotangent bundle of the supermanifold and $B$ is its underlying bundle, with just the bosonic coordinates. The $d \theta$ s are coordinates along the bundle, and unlike the case of the fibrewise integration presented above, they are bosonic. For that reason one needs $f$ to be sufficiently rapidly decreasing in them in order for the integral to converge. As will be demonstrated bellow, this is indeed the case for the super Fourier-Mukai transform.

\subsection{Review of the fermionic T-duality}

Consider the action (2.21) for the case when one has fermionic as well as bosonic variables, and $G_{i j}$ and $B_{i j}$ are graded-symmetric and graded -antisymmetric tensors respectively. Suppose that $G_{i j}$ and $B_{i j}$ do not depend on the fermionic variable $\theta^{1}$ [41]. Separating the variable $\theta^{1}$ one has

$$
S=\int d x^{+} d x^{-}\left(B_{11} \partial \theta^{1} \bar{\partial} \theta^{1}+E_{1 N} \partial \theta^{1} \bar{\partial} X^{N}+E_{M 1} \partial X^{M} \bar{\partial} \theta^{1}+E_{M N} \partial X^{M} \bar{\partial} X^{N}\right)
$$

Replacing derivatives of $\theta^{1}$ by fermionic vector $(A, \bar{A})$ and introducing a Lagrange multiplier field $\tilde{\theta}^{1}$ one gets

$$
S=\int d x^{+} d x^{-}\left(B_{11} A \bar{A}+E_{1 N} A \bar{\partial} X^{N}+E_{M 1} \partial X^{M} \bar{A}+E_{M N} \partial X^{M} \bar{\partial} X^{N}+\tilde{\theta}^{1}(\partial \bar{A}-\bar{\partial} A)\right)
$$

Integrating out $\tilde{\theta}^{1}$ imposes that

$$
A=\partial \theta^{1} \quad \text { and } \quad \bar{A}=\bar{\partial} \theta^{1} .
$$

Integrating out $(A, \bar{A})$ results in:

$$
\bar{A}=\frac{1}{B_{11}}\left((-)^{s_{M}} E_{1 M} \bar{\partial} X^{M}+\bar{\partial} \tilde{\theta}^{1}\right) \quad \text { and } \quad A=-\frac{1}{B_{11}}\left(E_{M 1} \partial X^{M}-\partial \tilde{\theta}^{1}\right)
$$


Inserting (4.6) in (4.4) one obtains fermionic T-dual background:

$$
\begin{aligned}
\tilde{B}_{11} & =-\frac{1}{B_{11}} \\
\tilde{E}_{1 M} & =\frac{E_{1 M}}{B_{11}} \\
\tilde{E}_{M 1} & =\frac{E_{M 1}}{B_{11}} \\
\tilde{E}_{M N} & =E_{M N}-\frac{E_{1 N} E_{M 1}}{B_{11}}
\end{aligned}
$$

or in the components:

$$
\begin{aligned}
\tilde{B}_{11} & =-\frac{1}{B_{11}} \\
\tilde{G}_{1 M} & =\frac{G_{1 M}}{B_{11}} \\
\tilde{B}_{1 M} & =\frac{B_{1 M}}{B_{11}} \\
\tilde{G}_{M N} & =G_{M N}-\frac{1}{B_{11}}\left(G_{1 N} B_{M 1}+B_{1 N} G_{M 1}\right) \\
\tilde{B}_{M N} & =B_{M N}-\frac{1}{B_{11}}\left(G_{1 N} G_{M 1}+B_{1 N} B_{M 1}\right)
\end{aligned}
$$

Equating (4.5) and (4.6) one gets:

$$
\partial \tilde{\theta}^{1}=B_{11} \partial \theta^{1}+E_{M 1} \partial X^{M} \quad \text { and } \quad \bar{\partial} \tilde{\theta}^{1}=B_{11} \bar{\partial} \theta^{1}-(-)^{s_{M}} E_{1 M} \bar{\partial} X^{M}
$$

The rest of the coordinates remains unchanged.

\subsection{Defects implementing the fermionic T-duality and the Super Poincare line bundle}

We now consider the action with defect, with target spaces related by the equations (4.7), and the defect given again by the correspondence space

$$
X^{N}=\tilde{X}^{N}, \quad N=2 \ldots \operatorname{dim} M
$$

and connection

$$
A=\theta^{1} d \tilde{\theta}^{1}
$$

with curvature

$$
F=d \theta^{1} \wedge d \tilde{\theta}^{1}
$$

We will call this super line bundle by analogy with the bosonic case a Super-Poincaré bundle. Now the defect equations of motion take the form:

$$
\begin{aligned}
E_{j 1} \partial X^{j}-(-)^{s_{j}} E_{1 j} \bar{\partial} X^{j}-\partial_{\tau} \tilde{\theta}^{1} & =0 \\
E_{j N} \partial X^{j}-(-)^{s_{j} s_{N}} E_{N j} \bar{\partial} X^{j}-\tilde{E}_{j N} \partial \tilde{X}^{j}+(-)^{s_{j} s_{N}} \tilde{E}_{N j} \bar{\partial} \tilde{X}^{j} & =0, \quad N=2 \ldots \operatorname{dim} M \\
\tilde{E}_{j 1} \partial \tilde{X}^{j}-(-)^{s_{j}} \tilde{E}_{1 j} \bar{\partial} \tilde{X}^{j}+\partial_{\tau} \theta^{1} & =0
\end{aligned}
$$


Additionally as before we have:

$$
\partial X^{N}+\bar{\partial} X^{N}=\partial \tilde{X}^{N}+\bar{\partial} \tilde{X}^{N}, \quad N=2 \ldots \operatorname{dim} M
$$

Solving (4.13), (4.14), (4.15), (4.16) we obtain

$$
\begin{array}{rlrl}
\bar{\partial} \tilde{X}^{N} & =\bar{\partial} X^{N}, & N=2 \ldots \operatorname{dim} M \\
\partial \tilde{X}^{N} & =\partial X^{N}, & N=2 \ldots \operatorname{dim} M \\
\partial \tilde{\theta}^{1} & =B_{11} \partial \theta^{1}+E_{M 1} \partial X^{M} & & \\
\bar{\partial} \tilde{\theta}^{1} & =B_{11} \bar{\partial} \theta^{1}-(-)^{s_{M}} E_{1 M} \bar{\partial} X^{M} &
\end{array}
$$

The details of the calculation can be found in appendix D. The relations (4.17) coincide with the equations (4.9). Therefore the defect given by the Super-Poincare bundle on the super-correspondence space induces the fermionic T-duality.

One can check that equations (4.8) and (4.17) imply:

$$
T=G_{i j} \partial X^{i} \partial X^{j}=\tilde{T}=\tilde{G}_{i j} \partial \tilde{X}^{i} \partial \tilde{X}^{j}
$$

and

$$
\bar{T}=G_{i j} \bar{\partial} X^{i} \bar{\partial} X^{j}=\tilde{\bar{T}}=\tilde{G}_{i j} \bar{\partial} \tilde{X}^{i} \bar{\partial} \tilde{X}^{j}
$$

which means that the defect is topological.

All this again can be generalized to the T-dualizing of several coordinates. Suppose we T-dualize the first $n$ coordinates, indexed by Greek letters.

The transformed background has the form

$$
\tilde{E}=\left(\begin{array}{cc}
-E_{\alpha \beta}^{-1} & E_{\alpha \beta}^{-1} E_{\beta N} \\
E_{M \alpha} E_{\alpha \beta}^{-1} & E_{M N}-E_{\beta N} E_{M \alpha} E_{\alpha \beta}^{-1}
\end{array}\right)
$$

Now we should consider the defect with the worldvolume

$$
X^{N}=\tilde{X}^{N}, \quad N=n+1 \ldots \operatorname{dim} M
$$

and connection

$$
A=\sum_{\alpha=1}^{n} \theta^{\alpha} d \tilde{\theta}^{\alpha}
$$

It has the curvature

$$
F=\sum_{\alpha=1}^{n} d \theta^{\alpha} \wedge d \tilde{\theta}^{\alpha}
$$

In the same way as above we can show that for $M$ and $\tilde{M}$ related by equations (4.20) this defect is topological and implies the defect equations of motion:

$$
\begin{aligned}
\bar{\partial} \tilde{X}^{N} & =\bar{\partial} X^{N} \\
\partial \tilde{X}^{N} & =\partial X^{N} \\
\partial \tilde{\theta}^{\alpha} & =E_{\beta \alpha} \partial \theta^{\beta}+E_{M \alpha} \partial X^{M} \\
\bar{\partial} \tilde{\theta}^{\alpha} & =E_{\alpha \beta} \bar{\partial} \theta^{\beta}-(-)^{s_{M}} E_{\alpha M} \bar{\partial} X^{N}
\end{aligned}
$$

We have obtained again T-duality relations for several T-dualized fermionic coordinates. 


\subsection{Super Fourier-Mukai transform}

We now elaborate the Fourier-Mukai transform for fermionic T-duality. It has the form:

$$
e^{-\hat{B}} \hat{\mathcal{G}}=\int d \eta e^{-B} \mathcal{G} e^{\eta \tilde{\eta}}
$$

with $\mathcal{G}$ and $B$ as in (2.54), where we set $\eta=d \theta^{1}$. As we explained $\eta$ is a bosonic variable, so we have a usual integration over $\eta$. From (4.8) one obtains:

$$
\begin{aligned}
\hat{B}-B= & -\frac{1}{2 B_{11}} \tilde{\eta}^{2}-\frac{1}{2} B_{11} \eta^{2} \\
& -\frac{1}{2 B_{11}}\left(G_{1 N} G_{M 1}+B_{1 N} B_{M 1}\right) d X^{M} d X^{N}+\frac{B_{1 M}}{B_{11}} \tilde{\eta} d X^{M}-B_{1 M} \eta d X^{M}
\end{aligned}
$$

Suppose that $\mathcal{G}$ does not depend on $\eta$. Using the formula for the Gaussian integral

$$
\int d x e^{-\frac{1}{2} a x^{2}+J x}=\frac{\sqrt{2 \pi}}{\sqrt{a}} e^{\frac{J^{2}}{2 a}}
$$

we obtain that the terms in (4.25) containing $B_{1 M}$ and the first quadratic term are canceled and, we end up with

$$
\hat{\mathcal{G}}=\frac{\sqrt{2 \pi}}{\sqrt{B_{11}}} \mathcal{G} e^{-\frac{1}{2 B_{11}} G_{1 N} G_{M 1} d X^{M} d X^{N}}
$$

Note that $G_{1 N}$ and $B_{1 N}$ have parity $(-)^{s_{N}+1}$. Hence if $d X^{M}$ and $d X^{N}$ are differentials of the bosonic coordiantes, the product $G_{1 N} G_{M 1}$ contains fermionic coordinates and drops if we consider the lowest $\theta=0$ components, in agreement with the observation [41] that the fermionic T-duality does not modify D-brane dimensionality. Note that the lowest $\theta=0$ components of (4.28) coincide with the homogeneous part of the transformation of the Ramond-Ramond forms in [41].

Using the transformations rules (4.20) equation (4.28) can be generalized to the case of the T-dualization of several fermionic variables $\theta^{\alpha}$. Keeping in mind that eventually we are going to project to the $\theta=0$ component we can set $G_{\alpha \beta}=0$, since $G_{\alpha \beta}=\eta^{a b} E_{a}^{\alpha} E_{b}^{\beta}$, and taking into account that $a$ and $b$ are bosonic and $\alpha$ and $\beta$ are fermionic, one sees that $E_{a}^{\alpha}$ and $E_{b}^{\beta}$ are odd. With this simplification the Fourier-Mukai transform for $\mathcal{G}$ independent on $\theta^{\alpha}$ can be computed to yield:

$$
\hat{\mathcal{G}}=\frac{\sqrt{2 \pi}}{\sqrt{\operatorname{det}\left\|B_{\alpha \beta}\right\|}} \mathcal{G} e^{-\frac{1}{2} B_{\alpha \beta}^{-1} G_{\alpha N} G_{M \beta} d X^{M} d X^{N}}
$$

The lowest component of (4.29) again coincides with the homogeneous part of the transformation of Ramond-Ramond forms in [41] for the fermionic T-dualization of the $n$ coordinates.

\section{Discussion}

It is shown in [57] that the generalization of the $\mathrm{SO}(d, d)$ duality group for the sigma models with a super target space is the orthosymplectic group $\operatorname{OSp}(d, d \mid 2 n)$. As in the bosonic 
case this group is generated by the superspace field redefinitions, the super B-field shift and the bosonic and fermionic dualities. Therefore the corresponding defects are given by the bosonic and fermionic dualities defects, constructed in sections 2.2 and 4.3 correspondingly, and the superspace analogue of the diagonal defects constructed in section 2.3. Some of these defects, as in the bosonic case considered in [29], can be non-invertible. Their study can lead to a new class of interfaces and is left for future work. The entries of the (semi)group of defects should be found from the analysis of the admissibility of the corresponding fields and angles of the defects.

It is also an interesting problem to find operator realization of the defect given by the Super-Poincaré line bundle implementing the fermionic T-duality.

Another open problem is to identify the possible connection between topological defects and the so called $\mathrm{T}$ folds [58] and generalize their construction.

\section{Acknowledgments}

The work of S. Elitzur is partially supported by the Israel Science Foundation Center of Excellence.

The work of E. Rabinovici is partially supported by the American-Israeli Bi-National Science Foundation and the Israel Science Foundation Center of Excellence.

The work of G. Sarkissian was partially supported by the Research project 11-1c258 of the State Committee of Science of Republic of Armenia and ANSEF hepth-2774 grant.

We would like also to thank Christoph Schweigert and Ingo Runkel for discussions.

\section{A Solution of the defect equations of motion in the bosonic case}

In subsection 2.2 we obtained the following defect equations of motion:

$$
\begin{aligned}
E_{j 1} \partial X^{j}-E_{1 j} \bar{\partial} X^{j}-\partial_{\tau} \tilde{X}^{1} & =0 \\
E_{j N} \partial X^{j}-E_{N j} \bar{\partial} X^{j}-\tilde{E}_{j N} \partial \tilde{X}^{j}+\tilde{E}_{N j} \bar{\partial} \tilde{X}^{j} & =0 \\
\tilde{E}_{j 1} \partial \tilde{X}^{j}-\tilde{E}_{1 j} \bar{\partial} \tilde{X}^{j}-\partial_{\tau} X^{1} & =0 . \\
\partial X^{N}+\bar{\partial} X^{N} & =\partial \tilde{X}^{N}+\bar{\partial} \tilde{X}^{N}
\end{aligned}
$$

The index $j$ runs from 1 to $\operatorname{dim} M$. The capital latin indices run from 2 to $\operatorname{dim} M$.

To solve these equations we perform the following steps.

Separating the first coordinate in (A.1) and (A.3) we obtain

$$
\begin{aligned}
E_{11}\left(\partial X^{1}-\bar{\partial} X^{1}\right)+E_{M 1} \partial X^{M}-E_{1 M} \bar{\partial} X^{M}-\partial \tilde{X}^{1}-\bar{\partial} \tilde{X}^{1} & =0 \\
-E_{11}\left(\partial X^{1}+\bar{\partial} X^{1}\right)-E_{M 1} \partial \tilde{X}^{M}-E_{1 M} \bar{\partial} \tilde{X}^{M}+\partial \tilde{X}^{1}-\bar{\partial} \tilde{X}^{1} & =0
\end{aligned}
$$

Taking sum and difference of (A.5) and (A.6) and taking into account (A.4) one gets

$$
\begin{aligned}
E_{11} \partial X^{1}-\partial \tilde{X}^{1}+E_{M 1} \partial \tilde{X}^{M}+G_{M 1}\left(\bar{\partial} \tilde{X}^{M}-\bar{\partial} X^{M}\right) & =0 \\
-E_{11} \bar{\partial} X^{1}-\bar{\partial} \tilde{X}^{1}-E_{1 M} \bar{\partial} \tilde{X}^{M}+G_{M 1}\left(\bar{\partial} \tilde{X}^{M}-\bar{\partial} X^{M}\right) & =0
\end{aligned}
$$


Separating the first coordinate in (A.2) and again using (A.4) we receive

$$
\begin{aligned}
& \frac{E_{1 N}}{E_{11}}\left(E_{11} \partial X^{1}-\partial \tilde{X}^{1}+E_{M 1} \partial \tilde{X}^{M}\right)+ \\
& -\frac{E_{N 1}}{E_{11}}\left(E_{11} \bar{\partial} X^{1}+\bar{\partial} \tilde{X}^{1}+E_{1 M} \bar{\partial} \tilde{X}^{M}\right)+2 G_{M N}\left(\bar{\partial} \tilde{X}^{M}-\bar{\partial} X^{M}\right)=0
\end{aligned}
$$

Combining (A.7), (A.8) and (A.9) finally we reach the equations

$$
\begin{array}{rlrl}
\bar{\partial} \tilde{X}^{N} & =\bar{\partial} X^{N} & N=2, \ldots \operatorname{dim} M \\
\partial \tilde{X}^{N} & =\partial X^{N} & N=2, \ldots \operatorname{dim} M \\
\partial \tilde{X}^{1} & =E_{11} \partial X^{1}+E_{M 1} \partial X^{M} & \\
\bar{\partial} \tilde{X}^{1}=-\left(E_{11} \bar{\partial} X^{1}+E_{1 M} \bar{\partial} X^{M}\right) &
\end{array}
$$

\section{B Geometry of the vector-axial duality defects for $\mathrm{SU}(2) / \mathrm{U}(1)$ cosets}

Here we provide details of the computation of the geometry of the defects considered in section 3. It is interesting to note that geometrically ( but not the flux and the symmetries! ) they coincide with some folded brane considered in [59], and one can use the results there. For reader convenience we collected the necessary stuff in this appendix.

$$
\left(g_{1}, g_{2}\right)=\left(C_{\mu} p, e^{i \alpha_{1} \frac{\sigma_{3}}{2}} p e^{i \alpha_{2} \frac{\sigma_{3}}{2}}\right)
$$

The conjugacy class is $C_{j}=l e^{\frac{2 \pi i j \sigma_{3}}{k}} l^{-1}$.

Equation (B.1) implies

$$
\operatorname{Tr}\left(g_{1} e^{i \alpha_{2} \frac{\sigma_{2}}{2}} g_{2}^{-1} e^{i \alpha_{1} \frac{\sigma_{2}}{2}}\right)=2 \cos \frac{2 j \pi}{k}
$$

Consider (B.2) as an equation in $\alpha_{1}$ and $\alpha_{2}$. The question is, which condition $g_{1}$ and $g_{2}$ should satisfy, in order that (B.2) has solutions in $\alpha_{1}$ and $\alpha_{2}$. To answer this question we introduce a new element $F=g_{1} e^{i \alpha_{2} \frac{\sigma_{2}}{2}} g_{2}^{-1}$ and analyze first for which $F$ there exists an $\alpha_{1}$ solving the equation

$$
\operatorname{Tr}\left(F e^{i \alpha_{1} \frac{\sigma_{2}}{2}}\right)=2 \cos \frac{2 j \pi}{k}
$$

Denoting the Euler coordinates of $F$ by $\theta_{F}, \tilde{\phi}_{F}$ and $\phi_{F}$, this equation takes the form

$$
\cos \theta_{F} \cos \left(\tilde{\phi}_{F}+\alpha_{1} / 2\right)=\cos \frac{2 j \pi}{k}
$$

eq. (B.4) has solution in $\alpha_{1}$ only if the inequality

$$
\cos 2 \theta_{F} \geq \cos \frac{4 j \pi}{k}
$$

is satisfied. Using the formula for the Euler angles of the product of two elements [60]

$$
\cos 2 \hat{\theta}=\cos 2 \theta_{1} \cos 2 \theta_{2}-\sin 2 \theta_{1} \sin 2 \theta_{2} \cos \left(\chi_{2}+\varphi_{1}\right)
$$


we obtain:

$$
\cos 2 \theta_{1} \cos 2 \theta_{2}+\sin 2 \theta_{1} \sin 2 \theta_{2} \cos \left(\varphi_{1}-\varphi_{2}+\alpha_{2}\right) \geq \cos \frac{2 j \pi}{k}
$$

For the inequality (B.7) to have solution in $\alpha_{2}$, the maximum value of the left hand side should be greater than $\cos \frac{2 j \pi}{k}$. The maximum value of the left hand side is $\cos 2\left(\theta_{1}-\theta_{2}\right)$. Therefore for (B.7) to have solutions the inequality

$$
\cos 2\left(\theta_{1}-\theta_{2}\right) \geq \cos \frac{4 j \pi}{k}
$$

should be satisfied.

Now we turn to the calculation of the geometry of the defect corresponding to the operator (3.47).

The matrix of the modular transformation of the SU(2) WZW model at the level $k$ is

$$
S_{\hat{j} j}^{\mathrm{SU}(2)}=\sqrt{\frac{2}{k+2}} \sin \left(\frac{(2 \hat{j}+1)(2 j+1)}{k+2}\right)
$$

In the large $-k$ limit the ratio of the $S$ matrix elements appearing in the defect operator simplifies to

$$
\frac{S_{\hat{j} j}^{\mathrm{SU}(2)}}{S_{0 j}^{\mathrm{SU}(2)}} \sim \frac{k}{\pi(2 j+1)} \sin [(2 j+1) \hat{\psi}]
$$

where we have introduced $\hat{\psi}=\frac{(2 \hat{j}+1) \pi}{k+2}$. To compute the overlap of the defect with the eigenposition state, we should remember that the coordinate wave functions of the parafermion theory are given by a gauge invariant wave function on $\mathrm{SU}(2)$. Gauge invariance means here that the wave functions are independent of the Euler angle $\phi$ or $\tilde{\phi}$ in the axially or vectorilally gauged models correspondingly. On the other hand wave functions on $\mathrm{SU}(2)$ are given by the normalized Wigner functions $\sqrt{2 j+1} D_{n m}^{j}$ [40]. Therefore in the axially gauged model the wave functions are $\sqrt{2 j+1} \mathcal{D}_{m,-m}^{j}$ and in the vectorially gauged model they are $\sqrt{2 j+1} \mathcal{D}_{m m}^{j}$. In the defect (3.47) only modes with $m=0$ are present. Remembering that $\mathcal{D}_{00}^{j}$ are the Legendre polynomials, finally we obtain at the large $k$ level:

$$
\left\langle\theta, \phi\left|Y_{\hat{j}}^{A B}\right| \tilde{\theta}, \tilde{\phi}\right\rangle=\sum_{j} \frac{k}{\pi} \sin [(2 j+1) \hat{\psi}] P_{j}(\cos 2 \theta) P_{j}(\cos 2 \tilde{\theta})
$$

where $P_{j}$ are the Legendre polynomials. Using the formula [60]:

$$
\begin{aligned}
& P_{j}\left(\cos \theta_{1}\right) P_{j}\left(\cos \theta_{2}\right)= \\
& \quad=\frac{1}{\pi} \int_{\left|\theta_{1}-\theta_{2}\right|}^{\theta_{1}+\theta_{2}} P_{j}(\cos \gamma) \frac{\sin \gamma d \gamma}{\sqrt{\left[\cos \gamma-\cos \left(\theta_{1}+\theta_{2}\right)\right]\left[\cos \left(\theta_{1}-\theta_{2}\right)-\cos \gamma\right]}}
\end{aligned}
$$

we obtain:

$$
\begin{aligned}
& \left\langle\theta, \phi\left|Y_{\hat{j}}^{A B}\right| \tilde{\theta}, \tilde{\phi}\right\rangle= \\
& =\frac{k}{\pi^{2}} \int_{|2 \theta-2 \tilde{\theta}|}^{2 \theta+2 \tilde{\theta}} \sum_{j} \sin [(2 j+1) \hat{\psi}] P_{j}(\cos \gamma) \frac{\sin \gamma d \gamma}{\sqrt{[\cos \gamma-\cos 2(\theta+\tilde{\theta})][\cos 2(\theta-\tilde{\theta})-\cos \gamma]}}
\end{aligned}
$$


Now let us evaluate [40]

$$
\sum_{j} \sin [(2 j+1) \hat{\psi}] P_{j}(\cos \gamma)=\frac{e^{i \hat{\psi}}}{2 i} \sum_{j} e^{(2 j) i \hat{\psi}} P_{j}(\cos \gamma)-\frac{e^{-i \hat{\psi}}}{2 i} \sum_{j} e^{-(2 j) i \hat{\psi}} P_{j}(\cos \gamma)
$$

Using the generating function for Legendre polynomials:

$$
\sum_{n} t^{n} P_{n}(x)=\frac{1}{\sqrt{1-2 t x+t^{2}}}
$$

one obtains for (B.14):

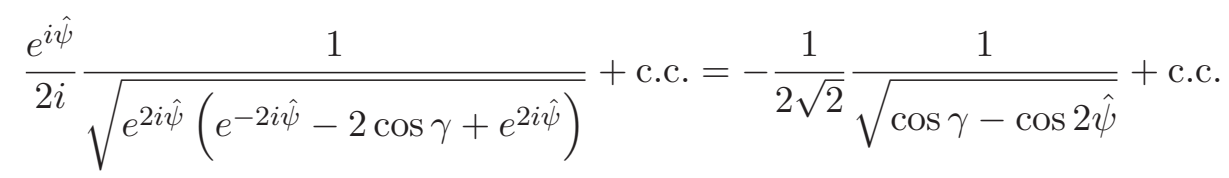

and introducing the Heavyside step function $\Theta$ one gets:

$$
\sum_{j} \sin [(2 j+1) \hat{\psi}] P_{j}(\cos \gamma) \sim \frac{\Theta(\cos \gamma-\cos 2 \hat{\psi})}{\sqrt{\cos \gamma-\cos 2 \hat{\psi}}}
$$

Inserting (B.17) in (B.13) one derives

$$
\begin{aligned}
& \left\langle\theta, \phi\left|Y_{\hat{j}}^{A B}\right| \tilde{\theta}, \tilde{\phi}\right\rangle \sim \\
& \sim \frac{k}{\pi^{2}} \int_{|2 \theta-2 \tilde{\theta}|}^{2 \theta+2 \tilde{\theta}} \frac{\Theta(\cos \gamma-\cos 2 \hat{\psi})}{\sqrt{\cos \gamma-\cos 2 \hat{\psi}}} \frac{\sin \gamma d \gamma}{\sqrt{[\cos \gamma-\cos 2(\theta+\tilde{\theta})][\cos 2(\theta-\tilde{\theta})-\cos \gamma]}}
\end{aligned}
$$

eq. (B.18) shows that the world-volume of the defect should satisfy the inequality

$$
\cos 2(\theta-\tilde{\theta}) \geq \cos 2 \hat{\psi}
$$

For $\hat{j}=0$ in the large $k$ limit it yields $\theta=\tilde{\theta}$.

\section{Some super geometry}

Here we review some of the definitions and results of super geometry, necessary for our needs, and in particular fibre-wise integration on super fibre bundles. We denote by $\Lambda(m)$ the exterior algebra in $m$ variables over a field $F$. This algebra is generated by an orthonormal basis $\theta_{1}, \ldots, \theta_{m}$ of $F^{m}$ and the relations $\theta_{i} \theta_{j}=-\theta_{j} \theta_{i}$.

Next we need to define the notion of a sheaf of objects in a category $\mathcal{C}$ on a space $X$. First, a presheaf, $\mathcal{F}$, of objects in a category $\mathcal{C}$ on a topological space $X$ is defined such that for every open set $U \subseteq X$ there is an object $\mathcal{F} \in \operatorname{obj}(\mathcal{C})$ and for every $V \subseteq U$ there is a morphism $r_{U, V} \in \operatorname{Mor}_{\mathcal{C}(\mathcal{F}(U), \mathcal{F}(U))}$, called restriction, with the following conditions:

1. $\mathcal{F}(\emptyset)=0(\emptyset$ being the null set, and 0 is a trivial object in $\operatorname{obj}(\mathcal{C}))$.

2. $r_{U, U}$ is the identity map between $\mathcal{F}(U)$ and itself.

3. For $W \subseteq V \subseteq U, r_{W, U}=r_{W, V} \circ r_{V, U}$. 
Now a sheaf is a presheaf with the following added conditions:

1. For any open set $U$ and a covering of it $U_{i}$, if $s \in \mathcal{F}(U)$ such that $r_{U, U_{i}}(s)=0$ for all $i$, then $s=0$.

2. For any open set $U$ and a covering of it $U_{i}$, if there exists elements $s_{i} \in \mathcal{F}\left(U_{i}\right)$ for any $i$, such that for any $i, j, r_{U_{i}, U_{i} \cap U_{j}}\left(s_{i}\right)=r_{U_{j}, U_{i} \cap U_{j}}\left(s_{j}\right)$, then there exists $s \in \mathcal{F}(U)$ such that $r_{U, U_{i}}=s_{i}$

One can verify that the algebra of $C^{\infty}$ function on a metric space $X$ is a sheaf on it.

For a metric space $X$, the sheaf of $C^{\infty}$ functions on $X$ is denoted by $\mathcal{O}_{X}$. The ring of functions restricted to a subset $U \subset X$ is denoted by $\mathcal{O}_{X}(U)$.

A smoothed superspace, $\mathcal{K}^{p, q}=\left(k^{p}, \mathcal{O}_{k^{p, q}}\right)$ is defined as a vector space $k^{p}$ with a sheaf $\mathcal{O}_{k^{p, q}}$ that is defined by $\mathcal{O}_{k^{p, q}}(U)=\mathcal{O}_{k^{p}}(U) \otimes \Lambda(q)$.

For a domain $U \subset k^{p}$ one defines a superdomain $\mathcal{U}^{p, q}=\left(U,\left.\mathcal{O}_{k^{p, q}}\right|_{U}\right) . U$ is called the underlying domain of $\mathcal{U}^{p, q}$. If $\left(x_{1}, \ldots, x_{p}\right)$ are the coordinates of $U$ and $\left(\theta_{1}, \ldots, \theta_{q}\right)$ are the generators of $\Lambda(q)$, we say that $\left(x_{1}, \ldots, x_{p}, \theta_{1}, \ldots, \theta_{q}\right)$ are the coordinates of $\mathcal{U}^{p, q}$.

A supermanifold, $\mathcal{M}$, is a ringed space $\left(M, \mathcal{O}_{\mathcal{M}}\right)$, where $\mathcal{O}_{\mathcal{M}}$ is a sheaf of commutative superalgebras and the following conditions are satisfied:

1. $M$ is a Hausdorf space with a countable base.

2. Every point $m$ in $M$ has a neighborhood $\mathrm{U}$, such that the ringed space $\left(U,\left.\mathcal{O}_{\mathcal{M}}\right|_{U}\right)$ is isomorphic to a superdomain $\mathcal{U}$.

A smooth function on a supermanifold can be written as

$$
f=\sum_{v} f_{v}(x) \theta^{v}
$$

Where $x=x_{1}, \ldots, x_{p}, v=v_{1}, \ldots v_{q}, v_{i} \in 0,1, \theta^{v}=\theta_{1}^{v_{1}} \cdot \ldots \cdot \theta_{q}^{v_{q}}$ and the sum is over all possible values of $v$. An integral of a function $f$ on a supermanifold $\mathcal{M}$ is defined as

$$
\int_{\mathcal{M}} f=\int_{M} f_{1,1, \ldots, 1}
$$

A derivative of a function of even and odd coordinates is defined as:

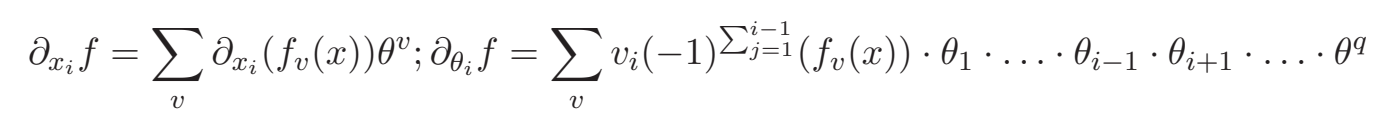

Note that a derivative with respect to the even variables are even (commuting), whereas that with respect to the grassmanian variables is odd. With this the tangent space at a point $m$ of (the underlying manifold $M$ of) a supermanifold $\mathcal{M}$ is the space spanned by the derivatives at $m$. As shown above, it is a super vector space of dimension $(p, q)$. It should be noted that a more rigorous definition of the tangent space exists, but it is very technical, and the definition used here suffices. Having defined the tangent space, the tangent bundle, $T \mathcal{M}$ is defined in the usual manner. It is a $(2 p, 2 q)$ dimensional supermanifold. 
More important to us than the tangent space is the cotangent space. It is a space derived from the tangent space by flipping the parity of all the generators. We denote the generators of the cotangent space by $d x_{1}, \ldots, d x_{p}, d \theta_{1}, \ldots, d \theta_{q}$. Note again that now the $d \theta$ 's are even, commuting variables, whereas the $d x$ 's are grassmanian. The cotangent bundle, denoted by $\Pi T M$ is, in a manner similar to the purely even case, a $(p+q, p+q)$ dimensional manifold.

A pseudodifferential form on a supermanifold $\mathcal{M}$ is a function on ПТ $M$. In a fashion akin to (C.1), such a function can be written as

$$
f=\sum_{v, u} f_{v, u}(x, d \theta) \theta^{v} d x^{u}
$$

Integration of a pseudodifferential form is defined just like in (C.2), and integrating only the variables along the cotangent space is called integration along a fibre, or fibrewise integration. Notice that the $d x$ 's are grassmanian and therefore pose no problem for the integration. The $d \theta$ 's, however, are even variables, and the fibre is a linear space, and so for the integral along the fibre to converge we need $f_{1,1, \ldots, 1}$ to be rapidly decreasing in those variables, i.e. for fixed $x$ that function decreases to zero faster than any polynomial in the $d \theta$ 's. In our analysis we use fibrewise integration when doing a "super Fourier-Mukai" transformation, and this condition would be satisfied.

\section{Solution of the defect equations of motion in the fermionic case}

In section 4 we obtained the defect equations of motion for the defect implementing fermionic T-duality:

$$
\begin{aligned}
E_{j 1} \partial X^{j}-(-)^{s_{j}} E_{1 j} \bar{\partial} X^{j}-\partial_{\tau} \tilde{\theta}^{1}=0 & \\
E_{j N} \partial X^{j}-(-)^{s_{j} s_{N}} E_{N j} \bar{\partial} X^{j}-\tilde{E}_{j N} \partial \tilde{X}^{j}+(-)^{s_{j} s_{N}} \tilde{E}_{N j} \bar{\partial} \tilde{X}^{j}=0, & N=2 \ldots \operatorname{dim} M \\
\tilde{E}_{j 1} \partial \tilde{X}^{j}-(-)^{s_{j}} \tilde{E}_{1 j} \bar{\partial} \tilde{X}^{j}+\partial_{\tau} \theta^{1}=0 & \\
\partial X^{N}+\bar{\partial} X^{N}=\partial \tilde{X}^{N}+\bar{\partial} \tilde{X}^{N}, & N=2 \ldots \operatorname{dim} M
\end{aligned}
$$

Writing separately terms with $\theta^{1}$ in (D.1) and with $\tilde{\theta}^{1}$ in (D.3) and using (4.7) we get

$$
\begin{aligned}
& E_{M 1} \partial X^{M}-(-)^{s_{M}} E_{1 M} \bar{\partial} X^{M}+B_{11}\left(\partial \theta^{1}+\bar{\partial} \theta^{1}\right)-\left(\partial \tilde{\theta}^{1}+\bar{\partial} \tilde{\theta}^{1}\right)=0 \\
& E_{M 1} \partial \tilde{X}^{M}-(-)^{s_{M}} E_{1 M} \bar{\partial} \tilde{X}^{M}-\left(\partial \tilde{\theta}^{1}+\bar{\partial} \tilde{\theta}^{1}\right)+B_{11}\left(\partial \theta^{1}+\bar{\partial} \theta^{1}\right)=0
\end{aligned}
$$

Taking sum and difference of (D.5) and (D.6) we obtain:

$$
\left(E_{M 1}+(-)^{s_{M}} E_{1 M}\right)\left(\bar{\partial} \tilde{X}^{M}-\bar{\partial} X^{M}\right)=0
$$

and

$$
E_{M 1} \partial \tilde{X}^{M}+B_{11} \partial \theta^{1}-\partial \tilde{\theta}^{1}-\left(\bar{\partial} \tilde{\theta}^{1}-B_{11} \bar{\partial} \theta^{1}+(-)^{s_{M}} E_{1 M} \bar{\partial} \tilde{X}^{M}\right)=0
$$

Separating terms with $\theta^{1}$ and $\tilde{\theta}^{1}$ also in (D.2) we obtain

$$
\begin{aligned}
& \frac{E_{1 N}}{B_{11}}\left(E_{M 1} \partial \tilde{X}^{M}+B_{11} \partial \theta^{1}-\partial \tilde{\theta}^{1}\right) \\
& +\frac{E_{N 1}(-)^{s_{N}}}{B_{11}}\left(\bar{\partial} \tilde{\theta}^{1}-B_{11} \bar{\partial} \theta^{1}+(-)^{s_{M}} E_{1 M} \bar{\partial} \tilde{X}^{M}\right)+\left(E_{M N}+(-)^{s_{M} s_{N}} E_{N M}\right)\left(\bar{\partial} \tilde{X}^{M}-\bar{\partial} X^{M}\right)=0
\end{aligned}
$$


Collecting all we get:

$$
\begin{aligned}
\bar{\partial} \tilde{X}^{N} & =\bar{\partial} X^{N}, & N=2 \ldots \operatorname{dim} M \\
\partial \tilde{X}^{N} & =\partial X^{N}, & N=2 \ldots \operatorname{dim} M \\
\partial \tilde{\theta}^{1} & =B_{11} \partial \theta^{1}+E_{M 1} \partial X^{M} & \\
\bar{\partial} \tilde{\theta}^{1} & =B_{11} \bar{\partial} \theta^{1}-(-)^{s_{M}} E_{1 M} \bar{\partial} X^{M} &
\end{aligned}
$$

Open Access. This article is distributed under the terms of the Creative Commons Attribution License which permits any use, distribution and reproduction in any medium, provided the original author(s) and source are credited.

\section{References}

[1] M. Oshikawa and I. Affleck, Boundary conformal field theory approach to the critical two-dimensional Ising model with a defect line, Nucl. Phys. B 495 (1997) 533 [cond-mat/9612187] [INSPIRE].

[2] V. Petkova and J. Zuber, Generalized twisted partition functions, Phys. Lett. B 504 (2001) 157 [hep-th/0011021] [INSPIRE].

[3] V. Petkova and J.-B. Zuber, Conformal field theories, graphs and quantum algebras, in MathPhys odyssey 2001: integrable models and beyond: in honor of Barry M. McCoy, M. Kashiwara and T. Miwa eds., (2002) [hep-th/0108236] [INSPIRE].

[4] V. Petkova and J. Zuber, The many faces of Ocneanu cells, Nucl. Phys. B 603 (2001) 449 [hep-th/0101151] [INSPIRE].

[5] C. Bachas, J. de Boer, R. Dijkgraaf and H. Ooguri, Permeable conformal walls and holography, JHEP 06 (2002) 027 [hep-th/0111210] [INSPIRE].

[6] J. Fuchs, I. Runkel and C. Schweigert, TFT construction of RCFT correlators 1. Partition functions, Nucl. Phys. B 646 (2002) 353 [hep-th/0204148] [INSPIRE].

[7] T. Quella and V. Schomerus, Symmetry breaking boundary states and defect lines, JHEP 06 (2002) 028 [hep-th/0203161] [INSPIRE].

[8] K. Graham and G. Watts, Defect lines and boundary flows, JHEP 04 (2004) 019 [hep-th/0306167] [INSPIRE].

[9] C. Bachas and M. Gaberdiel, Loop operators and the Kondo problem, JHEP 11 (2004) 065 [hep-th/0411067] [INSPIRE].

[10] J. Fröhlich, J. Fuchs, I. Runkel and C. Schweigert, Duality and defects in rational conformal field theory, Nucl. Phys. B 763 (2007) 354 [hep-th/0607247] [InSPIRE].

[11] T. Quella, I. Runkel and G.M. Watts, Reflection and transmission for conformal defects, JHEP 04 (2007) 095 [hep-th/0611296] [INSPIRE].

[12] C. Bachas and I. Brunner, Fusion of conformal interfaces, JHEP 02 (2008) 085 [arXiv:0712.0076] [INSPIRE].

[13] J. Fuchs, M.R. Gaberdiel, I. Runkel and C. Schweigert, Topological defects for the free boson CFT, J. Phys. A 40 (2007) 11403 [arXiv:0705.3129] [InSPIRE].

[14] J. Fuchs, C. Schweigert and K. Waldorf, Bi-branes: target space geometry for world sheet topological defects, J. Geom. Phys. 58 (2008) 576 [hep-th/0703145] [INSPIRE]. 
[15] I. Runkel and R.R. Suszek, Gerbe-holonomy for surfaces with defect networks, Adv. Theor. Math. Phys. 13 (2009) 1137 [arXiv:0808.1419] [INSPIRE].

[16] I. Brunner, H. Jockers and D. Roggenkamp, Defects and D-brane monodromies, Adv. Theor. Math. Phys. 13 (2009) 1077 [arXiv:0806.4734] [INSPIRE].

[17] G. Sarkissian and C. Schweigert, Some remarks on defects and T-duality, Nucl. Phys. B 819 (2009) 478 [arXiv:0810.3159] [InSPIRE].

[18] G. Sarkissian, Defects and permutation branes in the Liouville field theory, Nucl. Phys. B 821 (2009) 607 [arXiv:0903.4422] [InSPIRE].

[19] G. Sarkissian, Canonical quantization of the WZW model with defects and Chern-Simons theory, Int. J. Mod. Phys. A 25 (2010) 1367 [arXiv:0907.3395] [InSPIRE].

[20] V. Petkova, On the crossing relation in the presence of defects, JHEP 04 (2010) 061 [arXiv:0912.5535] [INSPIRE].

[21] J. Fröhlich, J. Fuchs, I. Runkel and C. Schweigert, Defect lines, dualities and generalised orbifolds, arXiv:0909.5013 [INSPIRE].

[22] N. Drukker, D. Gaiotto and J. Gomis, The virtue of defects in $4 D$ gauge theories and $2 D$ CFTs, JHEP 06 (2011) 025 [arXiv: 1003.1112] [INSPIRE].

[23] S. Fredenhagen, Boundary conditions in Toda theories and minimal models, JHEP 02 (2011) 052 [arXiv:1012.0485] [INSPIRE].

[24] A. Kapustin and K. Setter, Geometry of topological defects of two-dimensional $\sigma$-models, arXiv:1009.5999 [INSPIRE].

[25] G. Sarkissian, Defects in $G / H$ coset, $G / G$ topological field theory and discrete Fourier-Mukai transform, Nucl. Phys. B 846 (2011) 338 [arXiv:1006.5317] [InSPIRE].

[26] R.R. Suszek, Defects, dualities and the geometry of strings via gerbes. I. Dualities and state fusion through defects, arXiv:1101.1126 [INSPIRE].

[27] G. Sarkissian, Some remarks on D-branes and defects in Liouville and Toda field theories, Int. J. Mod. Phys. A 27 (2012) 1250181 [arXiv:1108.0242] [InSPIRE].

[28] Y. Satoh, On supersymmetric interfaces for string theory, JHEP 03 (2012) 072 [arXiv:1112.5935] [INSPIRE].

[29] C. Bachas, I. Brunner and D. Roggenkamp, A worldsheet extension of $O(d, d: Z)$, JHEP 10 (2012) 039 [arXiv: 1205.4647] [INSPIRE].

[30] J. Fuchs, C. Schweigert and A. Valentino, Bicategories for boundary conditions and for surface defects in $3 D$ TFT, arXiv: 1203.4568 [INSPIRE].

[31] D. Gaiotto, Domain walls for two-dimensional renormalization group flows, JHEP 12 (2012) 103 [arXiv:1201.0767] [INSPIRE].

[32] R.R. Suszek, Defects, dualities and the geometry of strings via gerbes. II. Generalised geometries with a twist, the gauge anomaly and the gauge-symmetry defect,

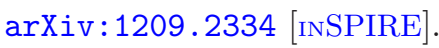

[33] N. Carqueville and I. Runkel, Orbifold completion of defect bicategories, arXiv:1210.6363 [INSPIRE].

[34] A. Giveon, M. Porrati and E. Rabinovici, Target space duality in string theory, Phys. Rept. 244 (1994) 77 [hep-th/9401139] [InSPIRE]. 
[35] K. Bardakci, E. Rabinovici and B. Saering, String models with $c<1$ components, Nucl. Phys. B 299 (1988) 151 [inSPIRE].

[36] W. Nahm, Gauging symmetries of two-dimensional conformally invariant models, UCD-88-02, (1988) [INSPIRE].

[37] D. Altschuler, K. Bardakci and E. Rabinovici, A construction of the $c<1$ modular invariant partition functions, Commun. Math. Phys. 118 (1988) 241 [INSPIRE].

[38] K. Gawędzki and A. Kupiainen, Coset construction from functional integrals, Nucl. Phys. B 320 (1989) 625 [inSPIRE].

[39] K. Bardacki, M. Crescimanno and E. Rabinovici, Parafermions from coset models, Nucl. Phys. B 344 (1990) 344 [inSPIRE].

[40] J.M. Maldacena, G.W. Moore and N. Seiberg, Geometrical interpretation of D-branes in gauged WZW models, JHEP 07 (2001) 046 [hep-th/0105038] [INSPIRE].

[41] N. Berkovits and J. Maldacena, Fermionic T-duality, dual superconformal symmetry and the amplitude/Wilson loop connection, JHEP 09 (2008) 062 [arXiv:0807.3196] [INSPIRE].

[42] P. Griffith and J. Harris, Principles of algebraic geometry, John Wiley \& Sons, New York U.S.A. (1978).

[43] T. Buscher, Path integral derivation of quantum duality in nonlinear $\sigma$-models, Phys. Lett. B 201 (1988) 466 [INSPIRE].

[44] A. Giveon, E. Rabinovici and G. Veneziano, Duality in string background space, Nucl. Phys. B 322 (1989) 167 [inSPIRE].

[45] S. Elitzur, A. Giveon, E. Rabinovici, A. Schwimmer and G. Veneziano, Remarks on non-Abelian duality, Nucl. Phys. B 435 (1995) 147 [hep-th/9409011] [INSPIRE].

[46] D. Huybrechts, Fourier-Mukai transforms in algebraic geometry, Oxford University Press, Oxford U.K. (2006).

[47] C. Bartocci, U. Bruzzo and D.H. Ruipérez, Fourier-Mukai and Nahm transform and applications in mathematical physics, Progr. Math. 276, Birkhäser, U.S.A. (2009).

[48] K. Hori, D-branes, T duality and index theory, Adv. Theor. Math. Phys. 3 (1999) 281 [hep-th/9902102] [INSPIRE].

[49] E. Bergshoeff, C.M. Hull and T. Ortín, Duality in the type-II superstring effective action, Nucl. Phys. B 451 (1995) 547 [hep-th/9504081] [INSPIRE].

[50] E. Eyras, B. Janssen and Y. Lozano, Five-branes, K K monopoles and T duality, Nucl. Phys. B 531 (1998) 275 [hep-th/9806169] [INSPIRE].

[51] R. Bott and L.W. Tu, Differential forms in algebraic topology, Graduate Texts in Mathematics 82, Springer-Verlag, New York U.S.A. (1982).

[52] I. Brunner and V. Schomerus, D-branes at singular curves of Calabi-Yau compactifications, JHEP 04 (2000) 020 [hep-th/0001132] [INSPIRE].

[53] J. Fuchs et al., Boundary fixed points, enhanced gauge symmetry and singular bundles on K3, Nucl. Phys. B 598 (2001) 57 [hep-th/0007145] [INSPIRE].

[54] J.N. Bernstein and D.A. Leites, How to integrate differential forms on supermanifolds, Funkts. Anal. Pril. 11 (1977) 70 [Funct. Anal. Appl. 11 (1977) 219]. 
[55] J.N. Bernstein and D.A. Leites, Integral forms and the Stokes formula on supermanifolds, Funkts. Anal. Pril. 11 (1977) 55 [Funct. Anal. Appl. 11 (1977) 45].

[56] P. Lavaud, Equivariant cohomology and localization formula in supergeometry, math.DG/0402068.

[57] P. Fré, P.A. Grassi, L. Sommovigo and M. Trigiante, Theory of superdualities and the orthosymplectic supergroup, Nucl. Phys. B 825 (2010) 177 [arXiv:0906.2510] [INSPIRE].

[58] C. Hull, A geometry for non-geometric string backgrounds, JHEP 10 (2005) 065 [hep-th/0406102] [INSPIRE].

[59] G. Sarkissian and M. Zamaklar, Symmetry breaking, permutation D-branes on group manifolds: boundary states and geometric description, Nucl. Phys. B 696 (2004) 66 [hep-th/0312215] [INSPIRE].

[60] N. Vilenkin, Special functions and the theory of group representations, American Mathematical Society (AMS), Providence U.S.A. (1968). 